Draft Version OCtober 25, 2018

Preprint typeset using $\mathrm{L}^{A} \mathrm{~T}_{\mathrm{E}} \mathrm{X}$ style emulateapj v. 11/10/09

\title{
RESOLVED IMAGES OF LARGE CAVITIES IN PROTOPLANETARY TRANSITION DISKS
}

\author{
Sean M. Andrews ${ }^{1}$, David J. Wilner ${ }^{1}$, Catherine Espaillat ${ }^{1,2}$, A. M. Hughes ${ }^{3}$, \\ C. P. Dullemond ${ }^{4}$, M. K. McClure ${ }^{5}$, Chunhua Qi ${ }^{1}$, And J. M. Brown ${ }^{1}$ \\ Draft version October 25, 2018
}

\begin{abstract}
Circumstellar disks are thought to experience a rapid "transition" phase in their evolution that can have a considerable impact on the formation and early development of planetary systems. We present new and archival high angular resolution $\left(0^{\prime \prime} 3 \approx 40-75 \mathrm{AU}\right)$ Submillimeter Array (SMA) observations of the $880 \mu \mathrm{m}(340 \mathrm{GHz})$ dust continuum emission from 12 such transition disks in nearby star-forming regions. In each case, we directly resolve a dust-depleted disk cavity around the central star. Using two-dimensional Monte Carlo radiative transfer calculations, we interpret these dust disk structures in a homogeneous, parametric model framework by reproducing their SMA continuum visibilities and spectral energy distributions. The cavities in these disks are large $\left(R_{\text {cav }}=15-73 \mathrm{AU}\right)$ and substantially depleted of small $(\sim \mu \mathrm{m}$-sized) dust grains, although their mass contents are still uncertain. The structures of the remnant material at larger radii are comparable to normal disks. We demonstrate that these large cavities are relatively common among the millimeter-bright disk population, comprising at least 1 in $5(20 \%)$ of the disks in the bright half (and $\geq 26 \%$ of the upper quartile) of the millimeter luminosity (disk mass) distribution. Utilizing these results, we assess some of the physical mechanisms proposed to account for transition disk structures. As has been shown before, photoevaporation models do not produce the large cavity sizes, accretion rates, and disk masses representative of this sample. It would be difficult to achieve a sufficient decrease of the dust optical depths in these cavities by particle growth alone: substantial growth (to meter sizes or beyond) must occur in large (tens of AU) regions of low turbulence without also producing an abundance of small particles. Given those challenges, we suggest instead that the observations are most commensurate with dynamical clearing due to tidal interactions with low-mass companions - young brown dwarfs or giant planets on long-period orbits. Subject headings: circumstellar matter — protoplanetary disks — planet-disk interactions — planets and satellites: formation — submillimeter: planetary systems
\end{abstract}

\section{INTRODUCTION}

Planets are made from gas and dust in disks that orbit young stars. The physical characteristics and evolution of that disk material have a profound impact on the outcome and efficiency of the planet formation process. While a massive theoretical effort has been made to elucidate the physical mechanisms that contribute to the assembly of a planetary system, any successful planet formation model needs to be informed by observations. An important goal is to generate models that incorporate empirical insights on the properties of circumstellar disks and are able to reproduce key demographic features of the exoplanet population (e.g., Ida \& Lin 2004, 2005; Alibert et al. 2005; Mordasini et al. 2009). One essential aspect of meeting that goal is the ability to make measurements related to the initial conditions imposed by the disk material. However, those constraints on initial conditions must be coupled with a characterization of disk evolution, which is ultimately responsible for the dynamical and physical properties of any resulting planetary system.

\footnotetext{
${ }^{1}$ Harvard-Smithsonian Center for Astrophysics, 60 Garden Street, Cambridge, MA 02138

2 NSF Astronomy \& Astrophysics Postdoctoral Fellow

3 Department of Astronomy, University of California at Berkeley, 601 Campbell Hall, Berkeley, CA 94720

4 Institut für Theoretische Astrophysik, Universität Heidelberg, Albert-Ueberle-Str. 2, Heidelberg, Germany 69120

${ }^{5}$ Department of Astronomy, University of Michigan, 830 Dennison Bldg, 500 Church Street, Ann Arbor, MI 48109
}

For most of its lifetime, the evolution of a protoplanetary disk is regulated by the interaction of gravitational and viscous stresses (e.g., Pringle 1981). Thought to be produced by magnetohydrodynamic turbulence (Balbus \& Hawlev 1991), the viscosity of the disk material acts to channel mass toward the star by transporting angular momentum out to larger disk radii (Lvnden-Bell \& Pringle 1974; Hartmann et al. 1998). This viscous evolution process decreases the densities and mass flow rates in the region of the disk where planets are made, and therefore sets a bound on the period where formation conditions are most favorable. Moreover, the viscous properties of the disk control the dynamics of solid bodies, from the radial drift of dust particles (Weidenschilling 1977a) to the orbital migration of protoplanets (Lin \& Papaloizou 1986). Hartmann et al. (1998) introduced some indirect methods for placing observational constraints on viscous evolution, based on tracking the decline of accretion rates with age (e.g., Sicilia-Aguilar et al. 2010) and characterizing disk sizes and surface density profiles with resolved millimeter observations (Kitamura et al. 2002; Andrews et al. 2009, 2010b; Isella et al. 2009, 2010a). More direct approaches have focused on characterizing the anomalous viscosities, including attempts to measure the disk magnetic field structure (Hughes et al. 2009b) and turbulent velocities (Hughes et al. 2011).

Although viscous evolution controls the long-term behavior of disk material, there is strong observational evidence that some (if not all) disks undergo a faster evolu- 
tionary phase that should have important consequences for the assembly and development of planetary systems. A small subsample of young disks exhibits a distinctive dip in their infrared spectral energy distribution (SED), suggesting that warm dust near the central star is substantially depleted compared to larger radii in the disk (Strom et al. 1989; Skrutskie et al. 1990). Since these so-called "transition" disks represent only a few percent of the total population in nearby star-forming regions with ages up to a few Myr (Muzerolle et al.|2010), the inside-out dispersal of disk material they appear to trace must occur quite rapidly (a few $\times 0.1 \mathrm{Myr}$, assuming all disks go through this phase at some point). A variety of dissipation mechanisms have been proposed to explain observations of transition disks, including extensive particle growth (Tanaka et al. 2005; Dullemond \& Dominik 2005), photoevaporative massloss in winds (Clarke et al. 2001; Alexander et al. 2006a), and tidal interactions with companions (e.g., Rice et al. 2003a; Ireland \& Kraus 2008). As a tantalizing example of the latter possibility, the transition disk structures might have been modified by dynamical interactions with very young giant planets (e.g., Bryden et al. 1999; Papaloizou et al. 2007). If that is the case, the size and material content of the dust-depleted region can in principle be used to constrain the orbit and mass of the unseen planetary companion (e.g., Lubow \& D'Angelo 2006; Varniére et al. 2006).

Regardless of which physical mechanism is responsible, this rapid evolutionary phase is potentially extraordinarily important in the context of planet formation. Since the short timescale for the dispersal or transformation of disk material can be quite influential on the accretion and migration history of young planets, this transitional period may play a dominant role in setting the final masses and orbital configurations of planetary systems. In that sense, the transition disks are crucial benchmarks for planet formation models that rely on observational comparisons of disk properties with the demographic characteristics of the much older population of exoplanets. There is an extensive literature on transition disk observations, ranging from their identification and initial probes of empirical trends (e.g., Naijita et al. 2007; Cieza et al. 2008; Kim et al. 2009; Merín et al. 2010) to detailed analyses of their broadband SEDs and infrared spectra (Calvet et al. 2002, 2005; D'Alessio et al. 2005; Espaillat et al. 2007, 2010, 2011). To facilitate the inclusion of this phase of disk evolution into the next generation of planet formation models, such studies must be coupled with firm observational constraints on the sizes and material contents of the dust-depleted zones in transition disks, as well as improved characterizations of their remnant mass reservoirs at larger radii. Those goals are best achieved by resolving their optically thin dust emission at millimeter wavelengths (Piétu et al. 2006; Brown et al. 2008, 2009; Hughes et al. 2009a).

In this paper, we present the first large sample of high angular resolution millimeter continuum observations of protoplanetary transition disks and analyze them in a homogeneous framework by constructing parametric models of their dust structures. These interferometric data and their calibration are described in $\S 2$. Detailed explanations of the adopted modeling formalism, radiative transfer calculations, and uncertainties in the modeling process are provided in $\S 3$. A preliminary characterization of the transition disk structures is included in $\S 4$. The data and models are synthesized and discussed in the context of disk evolution mechanisms in $\S 5$, with the key results summarized in $\S 6$. A brief commentary on each individual disk is included in the Appendix.

\section{OBSERVATIONS AND DATA REDUCTION}

We have constructed a sample of 12 nearby transition disks with high angular resolution $880 \mu \mathrm{m}$ continuum data using new and archival observations with the SMA interferometer (Ho et al. 2004). These targets were selected either based on the presence of a distinctive dip in their Spitzer InfraRed Spectrograph (IRS) spectrum (see the Appendix for references) and bright $880 \mu \mathrm{m}$ emission $\left(F_{\nu} \geq 100 \mathrm{mJy}\right)$ or the previous confirmation of a large dust-depleted cavity via direct imaging. Those criteria introduce some important biases that are discussed in more detail in $\S 4$. A journal of the SMA observations is provided in Table 1. Since some of the data in this sample were previously described elsewhere (Andrews et al. 2009, 2010b; Brown et al. 2008, 2009; Hughes et al. 2009a; Isella et al. 2010b), details of the adopted instrument configurations and calibration process will not be repeated here. New observations of DM Tau, LkCa 15, UX Tau, and RX J1615.3-3255 were obtained in 2010 February with the "very extended" (V) configuration of the SMA, with baseline lengths ranging from 68 to $509 \mathrm{~m}$. Those data were supplemented with shorter baseline observations in the compact ( $\mathrm{C}$ : baseline lengths of 6-70 m) and extended (E: baseline lengths of 28-226 m, excluding RX J1615-3255) configurations over the past year (since 2009 October), as were additional observations of GM Aur (C, E), LkH $\alpha 330$ (C), and MWC 758 (C).

For these recent observations (since fall of 2009), we used an expanded bandwidth capability (with the exception of the $\mathrm{C}$ observations of DM Tau) that separates each sideband into two intermediate frequency (IF) bands spanning $3-5 \mathrm{GHz}$ and $5-7 \mathrm{GHz}$ from the local oscillator (LO) frequency $(340.755 \mathrm{GHz}$, or $880 \mu \mathrm{m})$. Each IF band is composed of 24 partially overlapping $104 \mathrm{MHz}$ chunks, with the central chunk in the lower IF band split into 512 channels to sample the $\mathrm{CO} J=3-2$ transition $(345.796 \mathrm{GHz})$ at a resolution of $0.18 \mathrm{~km} \mathrm{~s}^{-1}$. All other chunks were divided into 32 coarse channels to measure the continuum. Using a cycle time of $\sim 10$ minutes, the observing sequence interleaved disk targets with nearby quasars: J0510+180, 3C 111, and 3C 120 for the northern disks and J1626-298, J1625-254, and J1604-446 for RX J1615-3255. When the targets were at low elevations $\left(<20^{\circ}\right)$, planets (Uranus, Mars), satellites (Titan, Callisto), and bright quasars (3C 273 and 3C 454.3) were observed as bandpass and absolute flux calibrators, depending on their availability and the array configuration. Weather conditions were excellent, with $225 \mathrm{GHz}$ atmospheric opacities below 0.1 (2.0 mm of precipitable water vapor) in all cases and substantially lower (down to 0.03 , or $0.6 \mathrm{~mm}$ of water vapor) for some observations.

The data were calibrated using the MIR software package, treating the individual IF bands separately to independently assess the accuracy of the process. The bandpass response was corrected based on observations of a bright quasar, and broadband continuum channels 
Table 1

SMA Observing Journal

\begin{tabular}{|c|c|c|c|c|c|c|}
\hline $\begin{array}{l}\text { Name } \\
(1)\end{array}$ & $\begin{array}{c}\alpha[\mathrm{J} 2000] \\
(2)\end{array}$ & $\begin{array}{c}\delta[\mathrm{J} 2000] \\
(3)\end{array}$ & $\begin{array}{l}\text { Region } \\
\text { (4) }\end{array}$ & $\begin{array}{c}\text { Array } \\
(5)\end{array}$ & $\begin{array}{l}\text { UT Date } \\
(6)\end{array}$ & $\begin{array}{c}\text { Reference } \\
(7)\end{array}$ \\
\hline MWC 758 & 053027.53 & +251956.9 & Tau? & $\mathrm{E}$ & $2008 \operatorname{Jan} 28$ & 1 \\
\hline SAO 206462 & 151548.43 & -370916.3 & Sco-OB2? & $\mathrm{V}$ & 2007 May 27 & 3 \\
\hline $\mathrm{LkH} \alpha 330$ & 034548.29 & +322411.9 & Per & $\mathrm{V}$ & 2006 Nov 19 & $\begin{array}{l}3 \\
4 \\
2\end{array}$ \\
\hline SR 21 & 162710.28 & -241912.8 & Oph & $\begin{array}{l}\mathrm{V} \\
\mathrm{S}\end{array}$ & $\begin{array}{l}2007 \text { Jun } 10 \\
2008 \text { Aug } 29\end{array}$ & $\begin{array}{l}3 \\
5\end{array}$ \\
\hline UX Tau A & 043004.00 & +18 1349.3 & Tau & $\begin{array}{l}\mathrm{C} \\
\mathrm{E} \\
\mathrm{V}\end{array}$ & $\begin{array}{l}2009 \text { Oct } 23 \\
2010 \text { Jan } 9 \\
2010 \text { Feb } 5\end{array}$ & $\begin{array}{l}2 \\
2 \\
2\end{array}$ \\
\hline SR $24 \mathrm{~S}$ & 162658.51 & -244537.0 & Oph & $\begin{array}{l}\mathrm{V} \\
\mathrm{C}\end{array}$ & $\begin{array}{l}2009 \text { Mar } 25 \\
2009 \text { May } 4\end{array}$ & $\begin{array}{l}6 \\
6\end{array}$ \\
\hline DoAr 44 & 163133.46 & -242737.4 & Oph & $\begin{array}{l}\mathrm{C} \\
\mathrm{V}\end{array}$ & $\begin{array}{l}2008 \text { May } 13 \\
2008 \text { Apr } 3\end{array}$ & $\begin{array}{l}5 \\
5\end{array}$ \\
\hline $\mathrm{LkCa} 15$ & 043917.79 & +222103.2 & Tau & $\begin{array}{l}\mathrm{C} \\
\mathrm{E} \\
\mathrm{V}\end{array}$ & $\begin{array}{l}2009 \text { Oct } 23 \\
2010 \text { Jan } 13 \\
2010 \text { Feb } 6\end{array}$ & $\begin{array}{l}2 \\
2 \\
2\end{array}$ \\
\hline RX J1615-3255 & 161520.20 & -325505.1 & Lup & $\begin{array}{l}\mathrm{V} \\
\mathrm{C}\end{array}$ & $\begin{array}{l}2010 \text { Feb } 16 \\
2010 \text { May } 1\end{array}$ & $\begin{array}{l}2 \\
2\end{array}$ \\
\hline GM Aur & 045510.98 & +302159.3 & Tau & $\begin{array}{l}\mathrm{V} \\
\mathrm{C} \\
\mathrm{E}\end{array}$ & $\begin{array}{l}2005 \text { Nov } 5 \\
2009 \text { Nov } 6 \\
2010 \text { Jan } 16\end{array}$ & $\begin{array}{l}7 \\
8 \\
8\end{array}$ \\
\hline DM Tau & 043348.74 & +18 1009.7 & Tau & V & $\begin{array}{l}2010 \text { Feb } 17 \\
2010 \text { Sep } 21\end{array}$ & $\begin{array}{l}2 \\
2 \\
2\end{array}$ \\
\hline WSB 60 & 162816.51 & -243658.3 & Oph & $\begin{array}{l}\mathrm{C} \\
\mathrm{V}\end{array}$ & $\begin{array}{l}2008 \text { May } 13 \\
2008 \text { Apr } 3\end{array}$ & $\begin{array}{l}5 \\
5\end{array}$ \\
\hline
\end{tabular}

Note. - Col. (1): Name of host star (in order of the stellar spectral type; see $\S 3.3$ and Table 3). Cols. (2) \& (3): Disk center coordinates (see §2). Col. (4): Starforming region. Col. (5): SMA configuration; $\mathrm{V}=$ very extended (68-509 $\mathrm{m}$ baselines), $\mathrm{E}=$ extended $(28-226 \mathrm{~m}), \mathrm{C}=$ compact $(16-70 \mathrm{~m})$, and $\mathrm{S}=\operatorname{subcompact}(6-70 \mathrm{~m})$. Col. (6): UT date of observation. Col. (7): Original references for SMA data: [1] Isella et al. (2010b), [2] - this paper, [3] - Brown et al. (2009), [4] - Brown et al. (2008), [5] - Andrews et al. (2009), [6] - Andrews et al. (2010b), [7] - Hughes et al. (2009a), [8]

- Hughes et al. (in preparation).

in each sideband and IF band were generated by averaging the central $82 \mathrm{MHz}$ of each chunk (except the one containing the CO $J=3-2$ transition). The visibility amplitudes were set based on observations of planets or satellites, with a systematic uncertainty of $\sim 10 \%$. The antenna-based complex gain response of the system as a function of time was determined with reference to the nearest local quasar projected on the sky, typically 3C 111, J0510+180, or J1626-298. The other quasars observed in each track were used to check the quality of phase transfer. Based on that information, we estimate that the millimeter "seeing" generated by atmospheric phase noise and any baseline errors is small, $\leq 0^{\prime \prime} 1$. The calibrated visibilities from different IF bands, sidebands, and array configurations were found to be in excellent agreement and combined. Because of the time elapsed (4-5yr) between observations of GM Aur and $\mathrm{LkH} \alpha 330$, the new data were corrected for proper motion (Ducourant et al. 2005) before combining the visibilities. The CO line data will be presented elsewhere.

The combined continuum visibilities were Fourier inverted, deconvolved with the CLEAN algorithm, and restored with a synthesized beam using the MIRIAD software package. Maps of the $880 \mu \mathrm{m}$ emission toward each target are exhibited together in Figure 1, These images were synthesized to accentuate structures on small angular scales. Given the variety in the available Fourier
Table 2

Aperture Synthesis Image Statistics

\begin{tabular}{|c|c|c|c|c|c|c|}
\hline $\begin{array}{c}\text { Name } \\
(1)\end{array}$ & $\begin{array}{c}F_{\text {tot }} \\
{[\mathrm{Jy}]} \\
(2)\end{array}$ & $\begin{array}{c}F_{\text {rec }} \\
{[\mathrm{Jy}]} \\
(3)\end{array}$ & $\begin{array}{c}I_{\text {peak }} \\
{[\mathrm{mJy}]} \\
(4)\end{array}$ & $\begin{array}{c}\sigma \\
{[\mathrm{mJy}]} \\
(5)\end{array}$ & $\begin{array}{c}\theta_{b} \\
{\left[{ }^{\prime \prime}\right]} \\
(6)\end{array}$ & $\begin{array}{c}\mathrm{PA}^{\circ} \\
{\left[{ }^{\circ}\right]} \\
(7)\end{array}$ \\
\hline MWC 758 & 0.18 & 0.16 & 59 & 1.1 & $0.82 \times 0.55$ & 93 \\
\hline SAO 206462 & 0.62 & 0.34 & 54 & 3.5 & $0.50 \times 0.24$ & 9 \\
\hline $\mathrm{LkH} \alpha 330$ & 0.21 & 0.21 & 28 & 2.1 & $0.31 \times 0.27$ & 84 \\
\hline SR 21 & 0.40 & 0.26 & 59 & 2.6 & $0.42 \times 0.30$ & 18 \\
\hline UX Tau A & 0.15 & 0.13 & 38 & 1.5 & $0.31 \times 0.28$ & 52 \\
\hline SR $24 \mathrm{~S}$ & 0.55 & 0.25 & 68 & 1.8 & $0.37 \times 0.26$ & 14 \\
\hline DoAr 44 & 0.21 & 0.21 & 62 & 2.7 & $0.60 \times 0.38$ & 23 \\
\hline $\mathrm{LkCa} 15$ & 0.41 & 0.39 & 40 & 0.9 & $0.41 \times 0.32$ & 64 \\
\hline RX J1615-3255 & 0.43 & 0.30 & 76 & 2.8 & $0.52 \times 0.26$ & 16 \\
\hline GM Aur & 0.64 & 0.56 & 65 & 3.5 & $0.32 \times 0.27$ & 38 \\
\hline DM Tau & 0.21 & 0.15 & 47 & 1.3 & $0.47 \times 0.31$ & 33 \\
\hline (inset) & & 0.02 & 13 & 1.3 & $0.37 \times 0.21$ & 31 \\
\hline WSB 60 & 0.25 & 0.25 & 113 & 3.5 & $0.59 \times 0.39$ & 25 \\
\hline (inset) & & 0.20 & 67 & 3.8 & $0.44 \times 0.23$ & 23 \\
\hline
\end{tabular}

Note. - Col. (1): Name of host star. Col (2): Total integrated flux density. Col. (3): Recovered integrated flux density in the synthesized maps in Figure 1 Col. (4): Peak flux density (in mJy beam ${ }^{-1}$ ). Col. (5): RMS $(1 \sigma)$ noise level in a $10^{\prime \prime}$-wide square centered on the stellar position. Col. (6): Synthesized beam dimensions. Col. (7): Synthesized beam major axis orientation (east of north). 


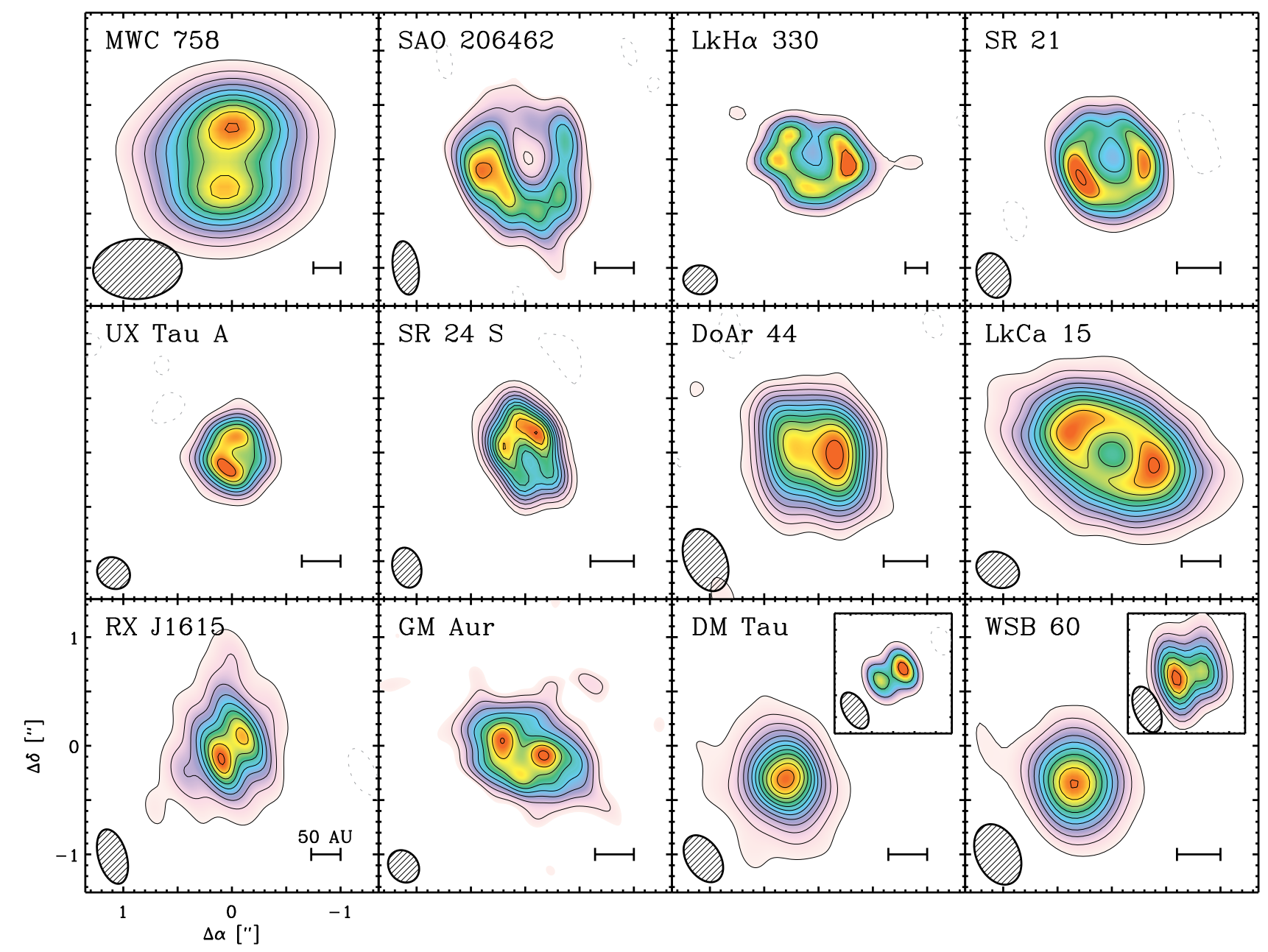

Figure 1. SMA aperture synthesis maps of the $880 \mu \mathrm{m}$ continuum emission from this sample of transition disks. Each panel is $22^{\prime \prime} 7$ on a side (offsets are referenced to the disk centers listed in Table 1 see $\$ 2$ ) and contains a 50 AU projected scale bar in the lower right for reference. Contours are drawn at $3 \sigma$ intervals, and the synthesized beam dimensions are marked in the lower left corner (RMS noise levels and beam dimensions are provided in Table 21). The inset images for the DM Tau and WSB 60 disks were synthesized with higher angular resolution, and are shown to scale.

coverage across the sample, a range of visibility weighting schemes were adopted to make the maps: the typical Briggs robust parameter values were 0-1, although in some cases uniform (DM Tau, WSB 60) or natural (SAO 206462) weighting schemes were used. The synthesized beam sizes are marked in the lower left of each panel and a projected $50 \mathrm{AU}$ scale bar is shown on the lower right. The disks located at southern declinations have elongated synthesized beams because their low observing elevations prevent sufficient long-baseline Fourier sampling in one dimension. The same is true for some northern sources that were scheduled for only a partial observing track (e.g., DM Tau). The image statistics are summarized in Table 2. including the total integrated flux densities, the flux densities recovered in the synthesized images in Figure 1 (note that $1-F_{\text {rec }} / F_{\text {tot }}$ indicates how much emission is spatially filtered for the adopted visibility weighting scheme), peak fluxes, noise levels, and beam dimensions. Each target exhibits the continuum morphology expected for a resolved emission "ring", with substantially reduced intensities near the stellar position. For intermediate viewing inclinations (or elongated synthesized beams), these limb-brightened rings display a distinctive double-peaked structure on either side of the stellar position. While that peak-to-peak separation offers a preliminary indication of the inner ring radius, more detailed modeling is required to accurately infer the disk structures.

With these unique $880 \mu \mathrm{m}$ emission morphologies, defining a disk center that can be used as a reference in such modeling can be problematic. Emission from the stellar photosphere is well below our detection limits at these long wavelengths, so there is no point-like reference in the data. We made an initial estimate of the disk centers based on the optical/near-infrared stellar positions, corrected for any known proper motion (Perrvman et al. 1997; Høg et al. 1998; Cutri et al. 2003; Ducourant et al. 2005). Aside from the multiple systems UX Tau and SR 24 (for which proper motion estimates are particularly uncertain), those positions were near the apparent emission centers in the synthesized $880 \mu \mathrm{m}$ images and well within the absolute SMA astrometric uncertainties $\left(\sim 00^{\prime \prime} 1\right)$. Those positions were then refined using a grid search that minimized the imaginary component of the visibilities (i.e., the position for which the RMS of the deviations from zero imaginary flux was smallest). In prin- 
ciple, the imaginary component of a symmetric source should be zero. Since our models will assume axisymmetric structures, this assumption for centering seems appropriate. An a posteriori comparison of the data and our modeling results (see $\S 4$ ) confirms that there is no statistically significant evidence for asymmetric emission structures on the angular scales probed by the SMA. The adopted reference centers are listed in Table 1 .

\section{MODELS OF TRANSITION DISK STRUCTURES}

The physical structures of transition disks are quite complex, and the few studies aimed at their characterization using spatially resolved data have adopted very different approaches (Piétu et al. 2006; Hughes et al. 2007, 2009a; Brown et al. 2008, 2009; Andrews et al. 2009, 2010a,b; Isella et al. 2010a, b). In this study, our goal is to analyze this large sample of transition disks in a homogeneous framework, emphasizing the new constraints on basic structural parameters that are responsible for the key features of the high angular resolution SMA data. These initial results should help guide more in-depth, individualized studies in the future. The modeling performed here is based on the previous work described by Andrews et al. (2009, 2010a, b) and Hughes et al. (2010), but includes some modifications meant to imitate (parametrically) the detailed treatments of Calvet et al. (2002, 2005), D'Alessio et al. (2005), and Espaillat et al. (2007, 2010). In the following sections, we describe a parametric model of the disk structure (§3.1), our assumptions about the dust populations (§3.2) and stellar properties (§3.3), and the radiative transfer calculations and our approach for comparing the models with observations (§3.4). Finally, we discuss the observable effects of the model parameters and important uncertainties (§3.5), and provide a concise summary of some key issues related to the modeling (§3.6) for those less interested in the details.

\subsection{Parametric Density Model}

The model structure includes two distinct radial zones: a dust-depleted "cavity" $\left(R \leq R_{\text {cav }}\right)$ and an "outer disk" reservoir $\left(R>R_{\text {cav }}\right)$. We define a global surface density profile, appropriate for a simple accretion disk with a power-law radial distribution of time-independent viscosities $\left(\nu \propto R^{\gamma}\right.$; see Lynden-Bell \& Pringle 1974; Hartmann et al. 1998),

$$
\Sigma_{g}=\Sigma_{c}\left(\frac{R}{R_{c}}\right)^{-\gamma} \exp \left[-\left(\frac{R}{R_{c}}\right)^{2-\gamma}\right]
$$

where $R_{c}$ is a characteristic scaling radius. This global profile is applied to the material in the outer disk: $\Sigma\left(R>R_{\text {cav }}\right)=\Sigma_{g}$. The densities in the cavity are scaled down by a constant depletion factor, $\delta_{\text {cav }}$, such that $\Sigma\left(R \leq R_{\text {cav }}\right)=\delta_{\text {cav }} \Sigma_{g}$. To facilitate a more homogeneous comparison of $\delta_{\text {cav }}$ values for a sample with a range of cavity sizes $\left(R_{\text {cav }}\right)$, we decomposed the cavity into a depleted "inner disk" of fixed extent (out to $R=10 \mathrm{AU}$ ) and an empty "gap" between $10 \mathrm{AU}$ and $R_{\text {cav }}$ (the arbitrary choice of the inner disk extent is discussed in $§ 3.5)$. Unless noted otherwise, the inner disk is truncated at the dust sublimation radius, $R_{\mathrm{sub}} \approx 0.07\left(L_{*} / \mathrm{L}_{\odot}\right)^{1 / 2} \mathrm{AU}$ (assuming $T_{\text {sub }}=1500 \mathrm{~K}$; Dullemond et al. 2001). Figure 2 a shows a generic surface density model for reference.
The vertical distribution of dust has been modified from our previous models, in an effort to more realistically treat the irradiation heating of the disk atmosphere. We have introduced a vertical gradient in the dust size distribution, such that larger grains are concentrated toward the midplane and smaller grains are distributed to larger vertical heights. In practice, this parameterization crudely mimics dust sedimentation (e.g., Dullemond \& Dominik 2004b; D'Alessio et al. 2006). At each radius, a population of "small" grains represents a small fraction of the total column density, $(1-f) \Sigma$, and is distributed vertically like a Gaussian with scale height $h=h_{c}\left(R / R_{c}\right)^{\psi}$. Conversely, most of the total column $(f \Sigma)$ is composed of a "large" grain population, condensed toward the midplane with a scale height $\chi h$ (where $\chi \leq 1$ ). In a spherical coordinate system with azimuthal and mirror symmetry, the two-dimensional density structures for each dust population are

$$
\begin{aligned}
& \rho_{s}=\frac{(1-f) \Sigma}{\sqrt{2 \pi} R h} \exp \left[-\frac{1}{2}\left(\frac{\pi / 2-\Theta}{h}\right)^{2}\right] \\
& \rho_{l}=\frac{f \Sigma}{\sqrt{2 \pi} R \chi h} \exp \left[-\frac{1}{2}\left(\frac{\pi / 2-\Theta}{\chi h}\right)^{2}\right]
\end{aligned}
$$

where the subscripts ' $s$ ' and ' $l$ ' denote the small and large dust populations, respectively, $\Theta$ is the (vertical) latitude coordinate measured from the pole $(\Theta=0)$ to the equator (the midplane, $\Theta=\pi / 2$ ), and the value of $\Sigma$ depends on the radial location as outlined above. Note that in this coordinate system, the scale height $h$ is an angle: the scale height in physical (distance) units is $H \approx h R$. A generic vertical density profile with this parametric form is shown in Figure $2 \mathrm{~b}$.

The distinctive infrared spectra of transition disks require small, but important, local modifications to the vertical structure near the disk edges at $R_{\text {sub }}$ and $R_{\text {cav }}$ (Natta et al. 2001; Calvet et al. 2002; Espaillat et al. 2010). At those locations, a large surface area of material is exposed directly (or with relatively low attenuation; see §3.5) to stellar irradiation. That exposure substantially heats a thin layer of material and "puffs" up the local dust distribution (Dullemond \& Dominik 2004a; D'Alessio et al. 2005). To mimic the emission signatures of these structures - referred to as the inner "rim" (at $R_{\text {sub }}$ ) and cavity "wall" (at $\left.R_{\text {cav }}\right)$ - we permit the local value of $h$ to be scaled up parametrically (to $h_{\text {rim }}$ and $h_{\text {wall }}$, respectively). Those perturbations are joined to the global scale height distribution exponentially over a small radial width (fixed here to $\Delta R=0.1 \mathrm{AU})$.

This structure model has 11 parameters: 5 describe the surface density profile, $\left\{\Sigma_{c}, \gamma, R_{c}, \delta_{\text {cav }}, R_{\text {cav }}\right\}$, and 6 others characterize the vertical distribution of dust, $\left\{h_{c}, \psi, \chi, f, h_{\text {rim }}, h_{\text {wall }}\right\}$. Some of these parameters can not be constrained with the available data, and were therefore fixed to representative values. The surface density gradient was kept at $\gamma=1$, in line with the results for "normal" disks with continuous dust distributions (Andrews et al. 2009, 2010b). We set the settling parameters to $\chi=0.2$ (the large grains are distributed to $20 \%$ of the scale height, $h$ ) and $f=0.85$ (85\% of the total column is composed of the large grain population). 

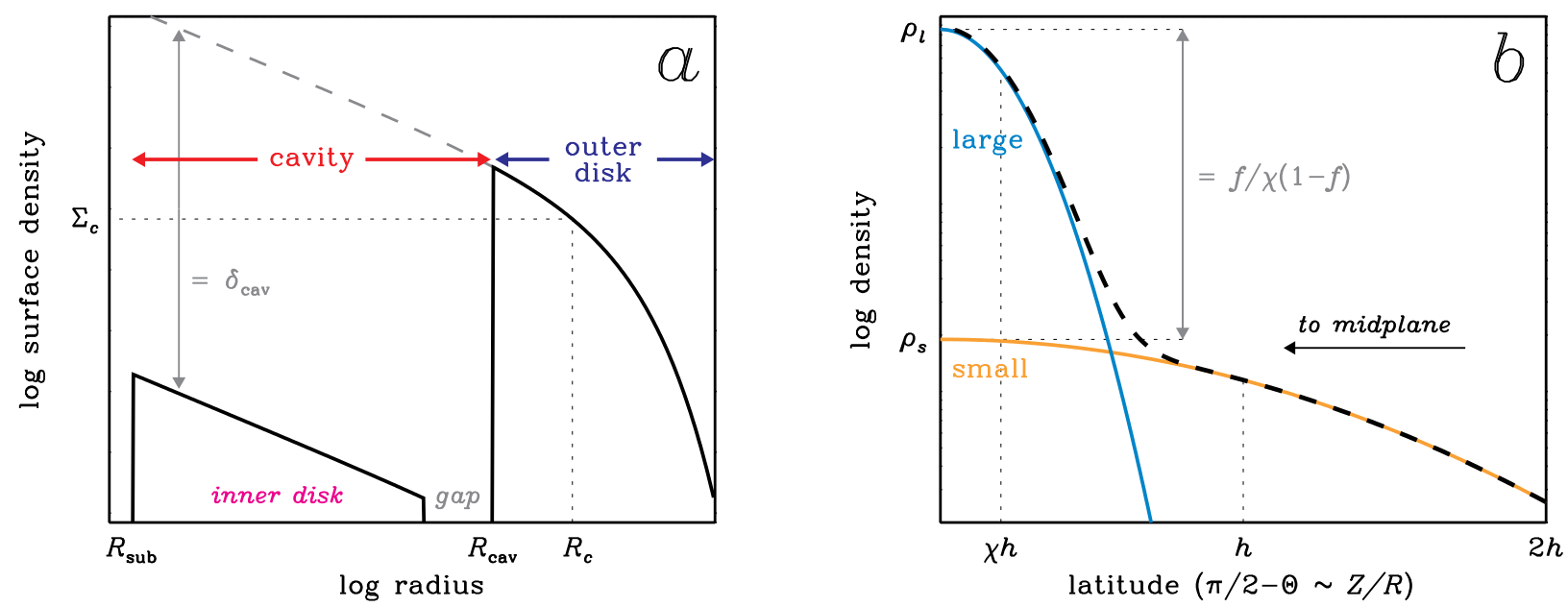

Figure 2. (a) A generic surface density model. The outer disk $\left(R>R_{\text {cav }}\right)$ follows the global $\Sigma_{g}$ profile, overlaid as a dashed curve. The surface densities in the cavity $\left(R \leq R_{\text {cav }}\right)$ are scaled down by a factor $\delta_{\text {cav }}$. The dust-depleted inner disk is truncated at the sublimation radius $\left(R_{\mathrm{sub}}\right)$ and extends outward to an arbitrary fixed radius $(10 \mathrm{AU})$. The gap between $10 \mathrm{AU}$ and $R_{\text {cav }}$ is assumed to be empty. (b) $\mathrm{A}$ generic vertical density profile. The blue curve tracks the "large" dust population, concentrated toward the midplane with a scale height $\chi h$ (in the models used here, $\chi$ is fixed to 0.2). The orange curve marks the "small" dust population that dominates in the disk atmosphere, and the dashed curve traces the composite profile. The fraction of the column density contributed by the large dust population is denoted $f$ (in the models used here, $f$ is fixed to 0.85 ).

The remaining parameters are allowed to freely vary, although substantial degeneracies remain (see §3.5).

\subsection{Dust Populations}

A parametric density structure must be populated with dust grains of a given size distribution and composition. For the sake of homogeneity, we assume dust grains with mineral abundances as in the interstellar medium (ISM) (see Weingartner \& Draine 2001), despite some evidence in individual cases for different compositions. Each dust population has a power-law distribution of sizes $(s), n(s) \propto s^{-p}$, from $s_{\min }=0.005 \mu \mathrm{m}$ to a specified $s_{\max }$. The dust properties in the outer disk are fixed: "small" grains have $s_{\max }=1 \mu \mathrm{m}$, "large" grains have $s_{\max }=1 \mathrm{~mm}$, and $p=3.5$. The opacity spectrum for a given size distribution was derived from Mie calculations. Since most of the mass is in "large" grains, the selection of $s_{\max } \sim \lambda=1 \mathrm{~mm}$ is significant because it tends to maximize the millimeter-wave dust opacity. Therefore, the densities inferred from the optically thin emission we have observed with the SMA can be considered lower bounds if much larger particles are present (D'Alessio et al. 2006; Draine 2006). The $880 \mu \mathrm{m}$ dust opacity for the large grain population is $3.6 \mathrm{~cm}^{2} \mathrm{~g}^{-1}$. The dust size distribution in the inner disk, inner rim, and cavity wall are assumed to be identical, but are characterized by free parameters for their size distribution, $\left\{s_{\max }, p\right\}$ (see $\S 3.4$ regarding the selection of these parameter values). Some representative opacity spectra are shown in Figure 3 .

\subsection{Stellar Properties}

Passive irradiation is the only heating mechanism considered here, and therefore the properties of the central star are pivotal in setting the thermal structure of a model. Careful selection of an input stellar spectrum is particularly important for modeling transition disks, as the infrared disk-to-star contrast is often low. Therefore, the details of the stellar spectrum can have a pronounced impact on how the small amount of dust inside the cavity

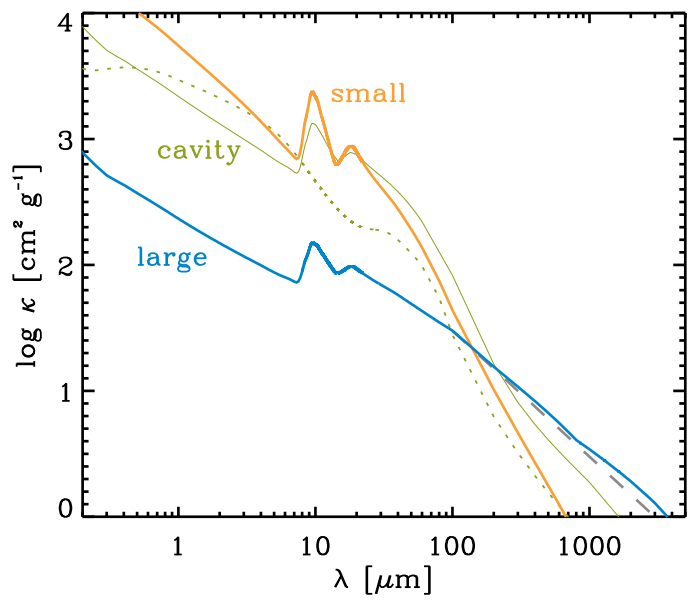

Figure 3. The opacity spectra for representative dust populations. The opacities for the small (orange) and large (blue) dust populations in the outer disk are fixed; the latter is similar to the canonical Beckwith et al. (1990) values (gray dashed line). As illustrative examples, the opacities for two different dust populations we have used inside the disk cavities are also shown (green): $\left\{s_{\max }\right.$, $p\}=\{10 \mu \mathrm{m}, 3.5\}$ (solid) and $\{1 \mu \mathrm{m}, 2.5\}$ (dotted).

is interpreted. To derive stellar input spectra, we first assigned effective temperature $\left(T_{\text {eff }}\right)$ values based on spectral classifications in the literature, using the conversions advocated by Kenyon \& Hartmann (1995) and Luhman (1999) for types earlier or later than M0, respectively. Luminosities $\left(L_{*}\right)$ were determined by matching reddened, scaled spectral synthesis models (Lejeune et al. 1997, for a fixed $T_{\text {eff }}$ ) to the optical/near-infrared photometry (see the Appendix). We utilized the extinction curve derived by McClure (2009), which at these wavelengths is identical to the Mathis (1990) law for a large total-to-selective extinction value, $R_{V}=5$. For cases without dynamical mass constraints, this process was iterated for different stellar masses $\left(M_{*}\right)$ estimated from the Siess et al. (2000) pre-main sequence models. The stellar parameters used to generate input spectra are compiled in Table 3 . 
Table 3

Stellar and Accretion Properties

\begin{tabular}{|c|c|c|c|c|c|c|c|c|c|c|}
\hline $\begin{array}{c}\text { Name } \\
(1) \\
\end{array}$ & $\begin{array}{l}\text { SpT } \\
(2) \\
\end{array}$ & $\begin{array}{l}T_{\text {eff }} \\
{[\mathrm{K}]} \\
(3) \\
\end{array}$ & $\begin{array}{l}A_{V} \\
(4) \\
\end{array}$ & $\begin{array}{c}d \\
{[\mathrm{pc}]} \\
(5)\end{array}$ & $\begin{array}{c}L_{*} \\
{\left[\mathrm{~L}_{\odot}\right]} \\
(6) \\
\end{array}$ & $\begin{array}{c}R_{*} \\
{\left[\mathrm{R}_{\odot}\right]} \\
(7) \\
\end{array}$ & $\begin{array}{c}M_{*} \\
{\left[\mathrm{M}_{\odot}\right]} \\
(8)\end{array}$ & $\begin{array}{c}\dot{M} \\
{\left[\mathrm{M}_{\odot} \mathrm{yr}^{-1}\right]} \\
(9) \\
\end{array}$ & $\begin{array}{c}\log L_{X} \\
{\left[\operatorname{erg~s}^{-1}\right]} \\
(10)\end{array}$ & $\begin{array}{l}\text { Ref } \\
(11) \\
\end{array}$ \\
\hline MWC 758 & A 8 & 7580 & 0.0 & 200 & 15 & 2.25 & 1.8 & $1 \times 10^{-8}$ & & $1,2,3$ \\
\hline SAO 206462 & F4 & 6590 & 0.3 & 140 & 7.8 & 2.15 & 1.6 & $6 \times 10^{-9}$ & 29.7 & $4,5,6,6$ \\
\hline $\mathrm{LkH} \alpha 330$ & G3 & 5830 & 3.1 & 250 & 15 & 3.75 & 2.2 & $2 \times 10^{-9}$ & $\ldots$ & $7,5,8, \quad \cdots$ \\
\hline SR 21 & G3 & 5830 & 6.3 & 125 & 10 & 3.15 & 2.0 & $<1 \times 10^{-9}$ & 30.0 & $9,5,10,11$ \\
\hline UX Tau A & G8 & 5520 & 1.9 & 140 & 3.5 & 2.05 & 1.5 & $1 \times 10^{-8}$ & 30.3 & $12,5,12,13$ \\
\hline SR $24 \mathrm{~S}$ & $\mathrm{~K} 2$ & 4990 & 7.0 & 125 & 4.0 & 2.70 & 2.0 & $1 \times 10^{-8}$ & 30.0 & $14,5,10,15$ \\
\hline DoAr 44 & K3 & 4730 & 2.2 & 125 & 1.4 & 1.75 & 1.3 & $9 \times 10^{-9}$ & 29.9 & $12,5,12,16$ \\
\hline $\mathrm{LkCa} 15$ & K3 & 4730 & 1.7 & 140 & 1.2 & 1.65 & 1.01 & $2 \times 10^{-9}$ & $<29.6$ & $12,17,18,19$ \\
\hline RX J1615-3255 & K5 & 4350 & 0.4 & 185 & 1.3 & 2.00 & 1.1 & $4 \times 10^{-10}$ & 30.4 & $20,5,5,21$ \\
\hline GM Aur & K5 & 4278 & 1.1 & 140 & 1.0 & 1.85 & 0.84 & $1 \times 10^{-8}$ & $<29.7$ & $22,23,18,19$ \\
\hline DM Tau & M1 & 3705 & 0.6 & 140 & 0.3 & 1.25 & 0.53 & $6 \times 10^{-9}$ & $<29.7$ & $24,17,18,25$ \\
\hline WSB 60 & M4 & 3270 & 3.7 & 125 & 0.2 & 1.40 & 0.25 & $2 \times 10^{-9}$ & $\cdots$ & $26,5,10, \quad \cdots$ \\
\hline
\end{tabular}

Note. - Col. (1): Name of host star. Col. (2): Spectral type. Col. (3): Visual extinction. Col. (4): Effective temperature. Col. (5): Luminosity. Col. (6): Estimated distance to star-forming region. Col. (7): Stellar radius. Col. (8): Stellar mass, determined either directly from CO spectral images or indirectly from pre-main sequence evolution models (see §3.2). Col. (9): Accretion rate. Col. (10): X-ray luminosity or $3 \sigma$ upper limit. Note that the value for UX Tau A may include contributions from the additional stars in the multiple system. To our knowledge, no X-ray measurements of WSB 60, $\mathrm{LkH} \alpha 330$, or MWC 758 have been published to date. Col. (11): Literature references for spectral classifications, $M_{*}, \dot{M}$, and $L_{X}$ : [1] - Beskrovnava et al. (1999), [2] Chapillon et al. (2008), [3] - Eisner et al. (2009), [4] - Dunkin et al. (1997), [5] - this paper (see §3.3), [6] - Grady et al. (2009), [7] - Cohen \& Kuhi (1979), [8] - Salyk et al. (2009), [9] - Prato et al. (2003), [10] - Natta et al. (2006), [11] - Grosso et al. (2000), [12] - Espaillat et al. (2010), [13] - König et al. (2001), [14] -Luhman \& Rieke (1999), [15] - Pillitteri et al. (2010), [16] - Montmerle et al. (1983), [17] - Piétu et al. (2007), [18] - Ingleby et al. (2009), [19] - Neuhauser et al. (1995), [20] - Wichmann et al. (1999), [21] - Krautter et al. (1997), [22] - White \& Ghez (2001), [23] - Dutrev et al. (1998), [24] Kenvon \& Hartmann (1995), [25] - Damiani et al. (1995), [26] - Wilking et al. (2005).

\subsection{Radiative Transfer and Modeling Procedure}

For a given set of parameters, a model structure was defined on a $200 \times 90$-cell grid in spherical coordinates, $\{R, \Theta\}$. The radial grid was logarithmically spaced from the sublimation radius to $1500 \mathrm{AU}$, with local refinements near $R_{\mathrm{sub}}, 10 \mathrm{AU}$ (the fixed edge of the inner disk), and $R_{\text {cav }}$ to sample the optical depth gradients at those locations with higher resolution. The latitude grid was linear, with three distinct resolution levels that depend on the vertical structure of the model: high near the condensed midplane, coarse at high latitudes near the pole, and intermediate in the disk atmosphere. Simulations of the stellar irradiation of that model density grid were conducted to determine the dust temperature structure, using the two-dimensional axisymmetric Monte Carlo radiative transfer code RADMC (see Dullemond \& Dominik 2004a). Those calculations explicitly accounted for the finite size of the star $\left(R_{*}\right.$; see Table 3 ) to properly treat any shadowing effects from the inner rim or cavity wall (stellar limb darkening is not considered). For a specified viewing geometry (a disk inclination, $i$, and major axis orientation, PA), a raytracing algorithm was used on the results of the radiative transfer calculations to compute a synthetic SED and set of $880 \mu \mathrm{m}$ continuum visibilities (sampled at the same spatial frequencies observed with the SMA).

Aided by a series of refined grid searches in parameterspace, this initial modeling effort was primarily conducted manually. A confirmation of fit quality was performed by comparing the synthetic data for a given parameter set with the observed SED and the deprojected, elliptically-averaged $880 \mu \mathrm{m}$ visibility profile (for details, see Andrews et al. 2009). For this model setup and such a large sample, parameter estimation with automated minimization algorithms would be computationally prohibitive: the free parameter-space is too large ( 8 density parameters, 2 dust parameters, and 2 viewing geometry parameters) and degenerate. Moreover, there are nontrivial technical obstacles: for example, great care is required in defining the model grid for a given parameter set, to ensure proper treatment of large optical depth gradients and small-scale features like the rim and wall (see also Andrews et al. 2009). Regardless of these challenges, good (albeit not unique, best-fit) matches to the data were found with a simple and systematic approach.

In most cases, the viewing geometry was fixed based on models of $\mathrm{CO}$ spectral images in the literature (Dutrev et al. 1998; Piétu et al. 2007; Isella et al. 2010b; Lyo et al. 2011) or the new $J=3-2$ data from the SMA (for LkH $\alpha 330$, UX Tau A, and RX J1615-3255; details will be presented elsewhere). No such information is available for the Ophiuchus disks (SR 21, SR $24 \mathrm{~S}$, DoAr 44, and WSB 60): estimates of $\{i, \mathrm{PA}\}$ in those cases were inferred from the aspect ratio and orientation of the continuum, and are considerably uncertain (see Andrews et al. 2009, 2010b). In practice, the cavity size, $R_{\text {cav }}$, was determined from the null position in the SMA visibility profile (see $\S 3.5$ ). The dust parameters in the cavity, $\left\{s_{\max }, p\right\}$, were set based on the strength of the $10 \mu \mathrm{m}$ silicate feature, and the density contrast $\left(\delta_{\text {cav }}\right)$ and rim height $\left(h_{\text {rim }}\right)$ were scaled to match the weak infrared excess from the inner disk. The height of the cavity wall 
$\left(h_{\text {wall }}\right)$ was adjusted to reproduce the shape and rise of the mid-infrared spectrum, and the scale height parameters, $\left\{h_{c}, \psi\right\}$, were modified based on the far-infrared SED. The characteristic radius, $R_{c}$, and density normalization, $\Sigma_{c}$, were inferred from the shape and amplitude of the $880 \mu \mathrm{m}$ visibility profile.

\subsection{Parameter Effects and Degeneracies}

To reinforce the intuition used to model these data and provide an overview of the individual parameter effects, it is helpful to decompose the disk structure into four essential elements: the inner rim, dust-depleted inner disk, cavity wall, and outer disk. Each element has a distinctive impact on the dust emission spectrum, and together they are capable of producing a remarkable diversity of observational signatures. To illustrate those signatures, we define a fiducial reference model and show separately the contributions from each structural element on the SED in Figure 4 (fiducial parameters are listed in the caption). Figure 5] explores how the SED and $880 \mu \mathrm{m}$ visibility profile respond to modest changes in the parameters of specific interest for transition disks: the rim and wall heights ( $h_{\text {rim }}$ and $h_{\text {wall }}$, respectively), the inner disk mass (set by $\left.\delta_{\text {cav }}\right)$, and the cavity size $\left(R_{\text {cav }}\right)$. The effects of more global structure parameters were discussed by Andrews et al. (2009).

The emission from the directly-illuminated dust in the inner rim dominates the near-infrared SED (see Figure 4). The luminosity of that hot component is set by the size (and optical depth) of the rim, such that a more "puffed-up" rim (larger $h_{\text {rim }}$ ) produces a brighter excess. A sufficiently high (and dense) rim can intercept enough starlight to shadow material at larger radii. The attenuated irradiation field that reaches those components produces less heating and therefore a fainter infrared spectrum (see Figure [5a). While this shadowing effect has been explored for normal disks (Dullemond 2000; Dullemond et al. 2001), its effects can be pronounced for transition disks because much of their infrared spectrum is produced by the directly-illuminated portion of the cavity wall (Espaillat et al. 2010). Espaillat et al. (2011) have shown that the Spitzer IRS spectra for many transition disks exhibit remarkable "see-saw" variability, such that an increase in the near-infrared emission is correlated with a decrease at mid-infrared wavelengths (and vice versa). They make a compelling argument that this variability is produced by changes in the height of the inner rim, as demonstrated in Figure $5 \mathrm{a}$ (see also Dullemond et al. 2003; Flaherty \& Muzerolle 2010).

Beyond the rim, the tenuous inner disk emits an infrared spectrum corresponding to the temperature range between $R_{\text {sub }}$ and $10 \mathrm{AU}$. In cases where this material is optically thin, the luminosity scales directly with the density (parameterized here by $\left.\delta_{\text {cav }}\right)$. However, small amounts of dust in the cavity can be optically thick. Even for substantial optical depths, the infrared luminosity still partly scales with density: for a given $h$, a denser disk has more dust at larger heights above the midplane, increasing the height and temperature of the infrared photosphere. The result is a brighter (and hotter) excess spectrum. The same is true for the inner rim, and therefore $\delta_{\text {cav }}$ can also have an impact on shadowing. Provided a sufficiently high rim height, $h_{\text {rim }}$ and $\delta_{\text {cav }}$ are degenerate (see Figure [5a,b): higher rims can compen-

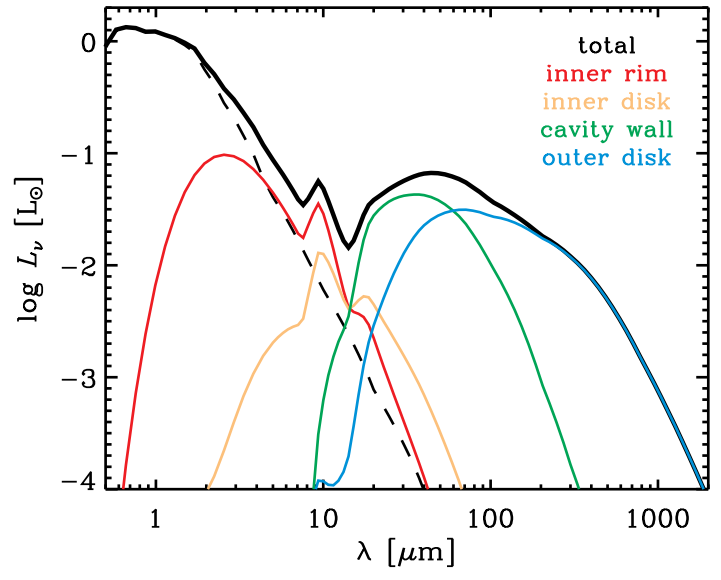

Figure 4. A fiducial model SED decomposed into individual structural elements. The ordinates are luminosity densities, $L_{\nu}=$ $4 \pi d^{2} \nu F_{\nu}$, in solar units. The model assumes a K3 star $(\sim 1 \mathrm{M} \odot$, $2 \mathrm{R} \odot$ ) irradiates a disk with a total mass $0.07 \mathrm{M} \odot, R_{c}=75 \mathrm{AU}$, and scale height distribution with $H_{100}=3 \mathrm{AU}$ and $\psi=0.2$. The densities inside $R_{\text {cav }}=40 \mathrm{AU}$ have been scaled down by a factor $\delta_{\text {cav }} \approx 10^{-6}$, and the inner rim and wall have been artificially puffed up a factor of $\sim 3$ over their local $H$ value $\left(H_{\text {sub }} \approx 0.005 \mathrm{AU}\right.$, or $0.5 R_{*}$, and $H_{\text {wall }} \approx 3 \mathrm{AU}$, respectively). The dust in the inner disk is the same as in the outer disk atmosphere, with $s_{\max }=1 \mu \mathrm{m}$ and $p=3.5$. The solid black curve is the sum of the colored curves and the contribution of the stellar photosphere (dashed).

sate for lower densities (at least partially). Moreover, the inner disk size (fixed here to $10 \mathrm{AU}$ ) is also degenerate with $\delta_{\text {cav }}$ : a more extended inner disk (a smaller gap, or even no gap) can usually be accomodated by decreasing $\delta_{\text {cav }}$. Other fixed assumptions about the inner disk structure can also have comparable effects (e.g., the width of the rim, shape of the density profile, local grain properties, etc.). The important point to be made is that even a small amount of ( $\sim \mu$ m-sized $)$ dust inside the cavity can have a substantial impact on the SED, depending rather sensitively on its detailed spatial distribution and grain properties. Therefore, without spatially resolved observations, the detailed characteristics of the material inside transition disk cavities are strongly model-dependent and highly uncertain.

Despite our ignorance of the detailed structures of the inner disk and rim, the cavity size can be measured from the SMA data with little ambiguity. Past efforts to indirectly infer $R_{\text {cav }}$ relied on estimating the wall temperature from the shape of the mid-infrared spectrum (e.g., D'Alessio et al. 2005; Calvet et al. 2005; Espaillat et al. 2007). Those models have become increasingly sophisticated to try and account for the irradiation gradients caused by shadowing (Espaillat et al. 2010), but still suffer from the implicit uncertainties in the inner disk properties (not to mention the detailed characteristics of the wall itself). However, a direct measurement of $R_{\text {cav }}$ is possible with a resolved, optically thin tracer like the millimeter continuum. The sharp emission contrast between the faint dust in the cavity and the bright material in the outer disk produces a clear "ring" morphology at $880 \mu \mathrm{m}$. The Fourier transform of that emission profile (i.e., the visibility profile) has distinctive nulls at discrete spatial frequencies, such that the location of the first null (where the real part of the visibilities change sign) is a relatively unambiguous measure of the inner edge of the ring $\left(R_{\text {cav }}\right.$; see Hughes et al. 2007). Figure 5r demonstrates the sensitivity of this null position to $R_{\text {cav }}$; mod- 

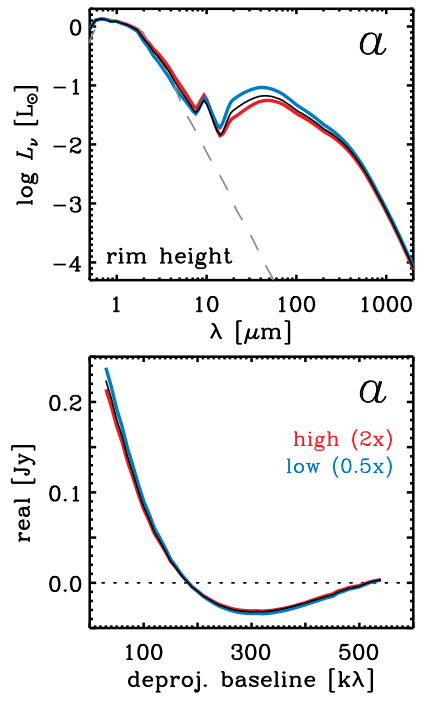
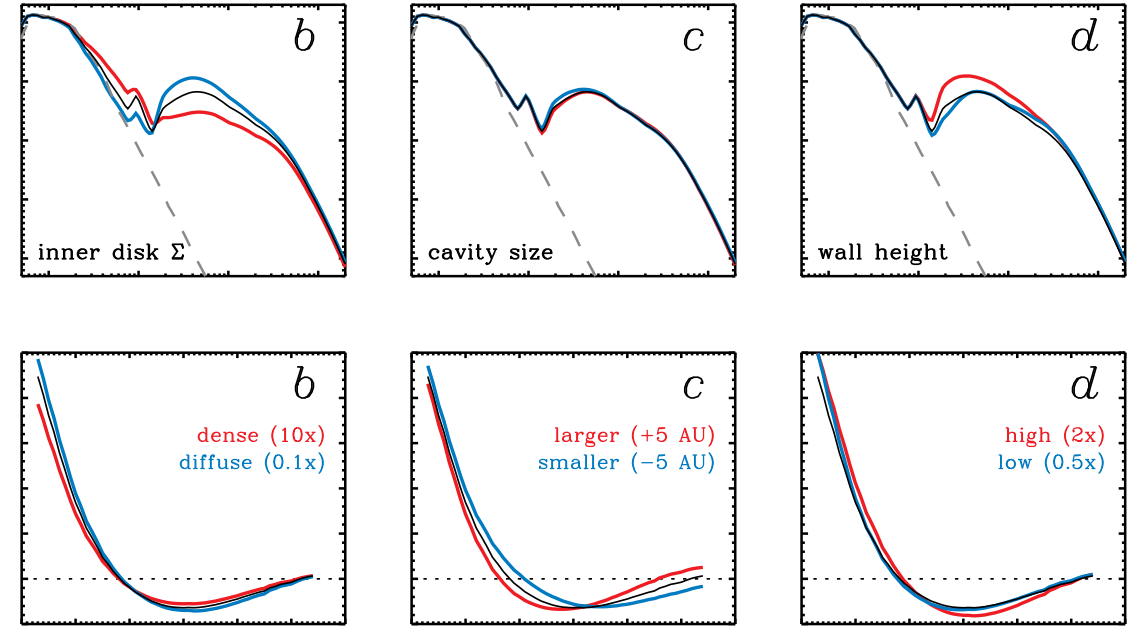

Figure 5. Effects of small modifications to key transition disk parameters on the SED (top) and $880 \mu \mathrm{m}$ visibility profile (bottom). The black curve is the fiducial model shown in Figure 4 The effects of parameter increases relative to the fiducial model are shown in red, decreases in blue: $(a)$ height of the inner rim $\left(h_{\text {rim }}\right)$, a factor of $2 ;(b)$ inner disk density $\left(1 / \delta_{\text {cav }}\right)$, a factor of $10 ;(c)$ cavity size $\left(R_{\text {cav }}\right)$, $\pm 5 \mathrm{AU}$; and $(d)$ cavity wall height $\left(h_{\text {wall }}\right)$, a factor of 2 .

ifications on the order of $10 \%$ are readily detectable for typical noise levels. However, we should note that these null positions also have a roughly linear dependance on the radial gradient of the surface brightness profile, which is set by the product of $\Sigma$ and the midplane temperature profile. Since the latter is approximately a power-law in these models $\left(T_{\text {mid }} \propto R^{-q}\right.$; typically $q \approx 0.5$ ), the $R_{\text {cav }}$ value inferred from a fixed null position is proportional to the sum $\gamma+q$. Since $\gamma=1$ is fixed in these models, we under-estimate the uncertainty on $R_{\text {cav }}$ : allowing $\gamma$ to vary by \pm 0.5 would contribute an additional $\sim 10-15 \%$ uncertainty to the cavity sizes.

In a simple model with an empty cavity, the directlyirradiated wall emits a thermal spectrum corresponding to the equilibrium temperature at its location $\left(R_{\text {cav }}\right)$ with a luminosity proportional to its vertical extent (Calvet et al. 2002; D'Alessio et al. 2005). To first order, the behavior expected from such a model is qualitatively reproduced in Figure 5d: smaller cavities have hotter walls and narrower infrared SED dips. As mentioned above, there are slight deviations in the details because shadowing can induce vertical temperature gradients in the wall. In principle, a high wall can shadow the outer disk if the dust is sufficiently settled (low $h_{c}$ and/or $\psi$ ). The detailed properties of the cavity wall (e.g., shape, thickness) are not well constrained by the SED alone; no current observations have sufficient resolution to isolate such a (presumably) small feature.

Although all disk modeling suffers from degeneracies between structure parameters and dust properties, it is important to explicitly highlight one key issue in these transition disk models. The standard mass-opacity degeneracy for optically thin emission (e.g., Beckwith \& Sargent 1991; Andrews \& Williams $2005 \mathrm{~b})$ and the intricate relationship between irradiation, heating, and grain properties in the disk atmosphere (D'Alessio et al. 1999, 2001, 2006; Chiang et al. 2001) are important, but will not be reviewed again here. The degeneracy of interest is related to how our method of inferring densities in the inner disk (i.e., $\delta_{\text {cav }}$ ) depends on the dust opacities inside the cavity. Since we lack any resolved millimeter signal inside $R_{\text {cav }}$ (although see $\S 4$ regarding $\mathrm{LkCa} 15$ ), the inner disk densities are determined solely from the infrared spectrum. In most cases, the relatively strong silicate emission features we observe suggest that the cavity is populated by "small" grains (i.e., $s_{\max } \approx 1-10 \mu \mathrm{m}$ or less). To be conservative, we do not assume that any additional material is present. This small dust becomes optically thick even at the relatively low column densities $\left(\sim 10^{-4} \mathrm{~g} \mathrm{~cm}^{-2}\right.$, give or take an order of magnitude; see Figure 31) that are present in some non-negligible part of the inner disk. The sizes of these high optical depth regions are effectively responsible for setting $\delta_{\text {cav }}$ : for a given structure, more mass would over-produce the infrared luminosities. In some sense, this aspect of the modeling tends to minimize $\delta_{\text {cav }}$ (or maximize the depletion of dust densities inside $R_{\text {cav }}$ ). However, more material could easily be accomodated in the inner disk if it has lower infrared opacities (e.g., $s_{\max }$ larger than a few $\mu \mathrm{m}$; see $\S 4$ ).

Finally, a few notes about the outer disk parameters are in order (for a more complete discussion, see Andrews et al. 2009). It is important to emphasize that the nature of transition disk structures makes it difficult to constrain radial gradient parameters $(\gamma$ or $\psi)$. For smaller disks, the same is true for the characteristic radius, $R_{c}$. The issue is limited dynamic range; only a few radial resolution elements can probe the millimeter emission in a ring (which is why we chose to fix $\gamma$ ), and the far-infrared SED that is less affected by the cavity is only sparsely sampled. While we have a reasonable constraint on the vertical distribution of dust $\left(h_{c}\right)$ from the infrared luminosities, the current lack of far-infrared data means that we do not have a good handle on the scale height gradient, $\psi$, for these transition disks with large cavities. Linking these models with scattered light observations that specialize in constraining the flaring angle (i.e., $\psi$ ) will be beneficial in the future.

Although they were fixed, the settling parameters $\{\chi$, $f\}$ merit a brief discussion. The fraction of large dust 
grains, $f$, helps set the height where stellar photons are absorbed: smaller $f$ means an increased optical depth (more abundant small grains) at a fixed height, and therefore a slightly warmer atmosphere that emits more infrared radiation. The effects of $\chi$, the fractional scale height of the large grain population, are subtle. In practice, $\chi$ modifies the vertical temperature gradient at intermediate heights in the disk atmosphere: a larger $\chi$ leads to a cooler temperature in a fixed vertical layer. Although potentially important for more direct tracers of the disk atmosphere (e.g., scattered light or molecular line emission), these effects are indistinguishable from adjustments to the scale height parameters, $\left\{h_{c}, \psi\right\}$, with the data used here.

\subsection{Modeling Synopsis}

The previous sections describe in detail an effort to model the SEDs and $880 \mu \mathrm{m}$ continuum visibilities for this sample of transition disks in a homogeneous, parametric framework. Before presenting those results, we first summarize some fundamental aspects of the modeling process:

- Using two-dimensional, axisymmetric Monte Carlo radiative transfer simulations, a parametric dust density structure is irradiated by a model star to determine an internally consistent temperature structure. The results of the simulation are used to generate synthetic data products to compare with observations. The underlying structure model is motivated by a simple viscous accretion disk model, with significant dust depletion in a central cavity.

- Given the relatively faint infrared excesses for transition disks, the adopted stellar input spectrum factors significantly into inferences about material inside the cavity.

- Small quantities of (small) dust in the cavity can produce large amounts of infrared emission. However, the lack of sufficient angular resolution in those regions means that the detailed structure of that material is unknown; considerable uncertainties remain for the mass content, spatial structure, and dust properties inside the cavity $\left(R \leq R_{\text {cav }}\right)$. Our estimates of density depletion tends to be maximal ( $\delta_{\text {cav }}$ is minimized): more material could reside in the inner disk if it has relatively lower infrared opacities (e.g., grain sizes larger than a few $\mu \mathrm{m})$.

- Small, local modifications to the vertical distribution of material near the sublimation radius (the inner rim) and outer edge of the disk cavity (the cavity wall) are needed to explain the distinctive infrared spectra of the transition disks. Because those same features can effectively shadow material at larger radii, their properties are at least partially entangled with one another as well as more global structure parameters.

- Because the dust-depleted cavities are spatially resolved at $880 \mu \mathrm{m}$, their sizes are determined directly and with reasonable accuracy (typically within $\pm 10 \%$ for a fixed $\gamma$ ).
- Inherent model degeneracies and limited observational information make it difficult to quantify several model parameters for an individual disk with sufficient confidence. However, because the modeling analysis was performed in a homogeneous framework for the entire sample, intra-sample (and qualitative) comparisons of the inferred structures are informative.

\section{RESULTS}

The estimated model parameters are compiled in Table 4. and the data are compared with the modeling results in Figures 6] and 7. Each row in those figures corresponds to an individual disk, with the panels showing (from left to right) images of the data (as in Figure 1), model (synthesized in the same way as the data), and residuals (the imaged residual visibilities), the SED (data in black, model in red, stellar input spectrum as dashed curve) and the $880 \mu \mathrm{m}$ visibility profile (data in black, model in red). In Table 4, we have recast $\Sigma_{c}$ into a total disk mass, $M_{d}$, by integrating the surface density profile and assuming a gas-to-dust mass ratio of 100:1. The scale height normalization $\left(h_{c}\right)$ is expressed in physical units at a fixed radius of $100 \mathrm{AU}$ for easy comparison. The inner rim and cavity wall heights are also expressed in physical units, the former in terms of the fraction of the stellar radius to suggest its potential for shadowing material at larger radii. The dust parameters correspond only to the material inside the cavity and wall (the dust populations in the outer disk were fixed; see $\S 3.2$ ): for reference, their values in the ISM are $s_{\max }=0.25 \mu \mathrm{m}$ and $p=3.5$ (Draine \& Lee 1984; Weingartner \& Draine 2001). The corresponding model surface density profiles are shown together in Figure 8, along with the underlying global profiles, $\Sigma_{g}$ (dashed; see Eq. 1). It is important to keep in mind that the surface density gradient was fixed, so all profiles have $\gamma=1$ by default. In practice, this means that the inner disk has $\Sigma \propto 1 / R$, while the outer disk is dominated by the exponential taper, $\Sigma \propto 1 / e^{R}$ (when $R_{c}>R_{\text {cav }}$, part of the outer disk $\Sigma$ profile flattens out to vary inversely with radius; e.g., GM Aur). The surface densities are also shown in an alternative "map" format in Figure 9, which better highlights the range of cavity sizes on a linear radius scale.

The transition disks in this sample have remarkably large dust-depleted cavities, with a range of radii $\left(R_{\text {cav }}\right)$ from 15-73 AU. The sample is likely biased toward large cavities, as they tend to produce a more obvious "dip" in the infrared SED. However, the extent of that bias is unclear, as some disks do not show any obvious signatures of a cavity in their infrared spectra. The WSB 60 and MWC 758 disks are good examples; their cavities were discovered serendipitously in millimeter-wave images (Andrews et al. 2009; Isella et al. 2010b). Efforts to infer $R_{\text {cav }}$ from the unresolved SED alone can be extraordinarily challenging (see $\S 3.5$ ). However, there have been some notable successes when compared with direct imaging, including GM Aur (Calvet et al. 2005; Hughes et al. 2009a), SAO 206462 (Brown et al. 2007, and this paper) and the older disk around TW Hya (Calvet et al. 2002; Hughes et al. 2007). While sophisticated SED modeling does surprisingly well in many cases - typically agreeing with the $R_{\text {cav }}$ values determined here within $\sim 30 \%$ 
Table 4

Model Parameters

\begin{tabular}{|c|c|c|c|c|c|c|c|c|c|c|c|c|}
\hline $\begin{array}{c}\text { Name } \\
\text { (1) }\end{array}$ & $\begin{array}{c}M_{d} \\
{\left[\mathrm{M}_{\odot}\right]} \\
(2)\end{array}$ & $\begin{array}{c}R_{c} \\
{[\mathrm{AU}]} \\
(3)\end{array}$ & $\begin{array}{c}H_{100} \\
{[\mathrm{AU}]} \\
(4)\end{array}$ & $\begin{array}{l}\psi \\
(5)\end{array}$ & $\begin{array}{c}R_{\text {cav }} \\
{[\mathrm{AU}]} \\
(6)\end{array}$ & $\begin{array}{c}\log \delta_{\text {cav }} \\
(7)\end{array}$ & $\begin{array}{c}H_{\mathrm{rim}} \\
{\left[\mathrm{R}_{*}\right]} \\
(8)\end{array}$ & $\begin{array}{c}H_{\text {wall }} \\
{[\mathrm{AU}]} \\
(9)\end{array}$ & $\begin{array}{l}s_{\max } \\
{[\mu \mathrm{m}]} \\
(10)\end{array}$ & $\begin{array}{c}p \\
(11)\end{array}$ & $\begin{array}{c}i \\
{\left[{ }^{\circ}\right]} \\
(12)\end{array}$ & $\begin{array}{l}\mathrm{PA} \\
{\left[{ }^{\circ}\right]} \\
(13)\end{array}$ \\
\hline MWC 758 & 0.008 & 25 & 18.3 & 0.05 & 73 & -5.3 & 4.6 & 9.5 & 0.25 & 3.5 & 23 & 49 \\
\hline SAO 206462 & 0.026 & 55 & 9.6 & 0.15 & 46 & -5.2 & 1.6 & 9.2 & 3 & 3.5 & 12 & 64 \\
\hline $\mathrm{LkH} \alpha 330$ & 0.024 & 60 & 6.5 & 0.20 & 68 & -5.2 & 4.1 & 6.8 & 1 & 2.5 & 35 & 80 \\
\hline SR 21 & 0.006 & 15 & 7.6 & 0.15 & 36 & -5.9 & 0.8 & 8.2 & 10 & 3.5 & 22 & 100 \\
\hline UX Tau & 0.007 & 20 & 4.1 & 0.35 & 25 & -5.7 & 0.5 & 0.6 & 1 & 2.5 & 35 & 176 \\
\hline SR $24 \mathrm{~S}$ & 0.045 & 40 & 4.5 & 0.20 & 29 & -5.4 & 3.2 & 1.5 & 0.25 & 3.5 & 45 & 24 \\
\hline DoAr 44 & 0.007 & 25 & 3.1 & 0.02 & 30 & -5.1 & 3.1 & 9.0 & 0.5 & 3.5 & 35 & 80 \\
\hline LkCa 15 & 0.055 & 85 & 2.9 & 0.20 & 50 & -6.0 & 1.0 & 7.0 & 0.25 & 3.5 & 49 & 241 \\
\hline RX J1615-3255 & 0.128 & 115 & 3.4 & 0.25 & 30 & -5.8 & $\ldots$ a & 2.0 & 0.25 & 3.5 & 41 & 143 \\
\hline GM Aur & 0.070 & 120 & 6.2 & 0.35 & 28 & -5.7 & 0.1 & 2.8 & 0.5 & 3.5 & 55 & 64 \\
\hline DM Tau & 0.040 & 135 & 4.2 & 0.20 & 19 & -4.8 & $\ldots \mathrm{a}$ & 5.7 & 3 & 3.5 & 35 & 155 \\
\hline WSB 60 & 0.028 & 30 & 10.6 & 0.10 & 15 & -1.7 & 0.4 & 0.8 & 1 & 3.5 & 28 & 117 \\
\hline
\end{tabular}

Note. - Col. (1): Name of host star. Col. (2): Total mass in the disk (gas+dust, assuming a gas-to-dust mass ratio of 100:1). Col. (3): Characteristic radius of the surface density profile. Col. (4): Scale height at $100 \mathrm{AU}$ (based on $h_{c}$ ). Col. (5): Power-law index of the radial scale height distribution. Col. (6): Radius of the dust-depleted disk cavity. Col. (7): Density contrast in the cavity, such that $\delta_{\text {cav }}=\Sigma / \Sigma_{g}$ when $R \leq R_{\text {cav }}$, where $\Sigma_{g}$ is the surface density profile for a continuous disk (see Equation 1 and §3.1). Col. (8): Scale height of the inner rim at the sublimation radius (expressed in units of the stellar radius). Col. (9): Scale height of the cavity wall. Col. (10): The maximum grain size for the dust population in the cavity and wall. Col. (11): The power-law index of the grain size distribution in the cavity and wall, such that $n(s) \propto s^{-p}$. Col. (12): Disk inclination $\left(0^{\circ}\right.$ is face-on). Col. (11): Position angle of the disk major axis, measured east of north.

a We have removed any dust out to a radius of $1 \mathrm{AU}$ for the DM Tau disk and $0.5 \mathrm{AU}$ for the RX J1615-3255 disk. Neither case requires a puffed-up inner rim to fit their infrared SED emission (see the Appendix).

(e.g., Brown et al. 2007; Espaillat et al. 2007) - there are also some cases where the predicted cavity sizes are much less consistent with the SMA images. The DM Tau disk is the most extreme example, where the $3 \mathrm{AU}$ cavity inferred by Calvet et al. (2005) is $\sim 6 \times$ smaller than measured here $\left(R_{\text {cav }}=19 \mathrm{AU}\right)$. Such large discrepancies are informative; they call for modifications to the assumed structure and/or dust properties inside the cavity. For example, Espaillat et al. (2011) were able to decrease their original estimate of the cavity size in the UX Tau A disk by more than a factor of 2 (from 71 to $30 \mathrm{AU}$; Espaillat et al. 2010) using an updated grain composition in the cavity wall, both improving their SED-based fit quality and providing better agreement with the resolved SMA data. These more extreme examples should serve as reminders that estimates of cavity sizes from SEDs are model-dependent; they should not be considered robust until the cavity is spatially resolved.

The apparent emptiness of the transition disk cavities is a striking aspect of the modeling results. Compared to the underlying global $\Sigma_{g}$ profiles (dashed curves in Figure 8), the surface densities inside these cavities have been decreased by $\sim 5-6$ orders of magnitude. The WSB 60 disk is a notable exception, where a more substantial amount of dust is required to account for the infrared excess around this cool star (the $\Sigma$ decrease is a factor of $\left.\sim 50, \delta_{\text {cav }}=0.02\right)$. In most cases, only a few percent of a lunar mass of dust $\left(\sim 10^{-10}-10^{-8} \mathrm{M}_{\odot}\right)$ is required to reproduce the observed infrared excesses. As explained in $§ 3.5$, the dust depletion factors $\left(\delta_{\text {cav }}\right.$; and therefore $\Sigma$ in the inner disk) were effectively determined from the infrared spectrum, and therefore are only relevant for small ( $\sim \mu$ m-sized) dust grains in the cavities. Increased densities (larger $\delta_{\text {cav }}$ ) could easily be accomodated for a population of larger dust grains that have lower infrared opacities (i.e., $s_{\max } \gg 1 \mu \mathrm{m}$ ). We can crudely estimate the mass of $\mathrm{mm}$-sized grains inside the cavities from the $880 \mu \mathrm{m}$ residual maps in Figures [6] and 7. Since our models produce no millimeter emission inside $R_{\text {cav }}$, the residual emission near the disk center should roughly trace the product of the dust opacity, inner disk mass, and a mean temperature $\left(F_{\nu} \propto \kappa M_{\text {cav }}\langle T\rangle\right.$; e.g., Andrews \& Williams 2005b). In most cases, those residuals are below the noise floor. Using $3 \sigma$ upper limits from the residual map centers (see Table 2), an $880 \mu \mathrm{m}$ opacity of $3.6 \mathrm{~cm}^{2} \mathrm{~g}^{-1}$ (see $\S 3.2$ ), and a mean temperature of $75 \mathrm{~K}$, the absence of significant residuals suggests that there is typically less than $\sim 1 \mathrm{M}_{\oplus}\left(\lesssim 10^{-6} \mathrm{M}_{\odot}\right)$ of mm-sized grains in these disk cavities 6 Due to our sensitivity limits, these $880 \mu \mathrm{m}$ constraints on the density contrasts are relatively weak $\left(\delta_{\text {cav }} \leq 0.01-0.1\right)$. The LkCa 15 disk is a remarkable exception, as it shows a significant $\left(\sim 6 \sigma ; F_{\nu} \approx 10 \mathrm{mJy}\right)$ and centralized residual peak that suggests a small reservoir $\left(\sim 1 \mathrm{M}_{\oplus}\right)$ of mm-sized grains are present inside its cavity.

Aside from the large dust-depleted cavities, these transition disks have surface density profiles similar to those inferred for normal disks (see Andrews et al. 2009, $2010 \mathrm{~b})$. Both populations exhibit the same range of characteristic scaling radii and disk masses, with $R_{c}=15$ -

6 Note that the constraint from the $880 \mu \mathrm{m}$ residuals in this simple example is technically less stringent than could be made from the infrared emission. The $1 \mu \mathrm{m}$ opacities for the large grain population, $\left\{s_{\max }=1 \mathrm{~mm}, p=3.5\right\}$, are only $\sim 50 \times$ lower than for $s_{\max }=1 \mu \mathrm{m}$ (see Figure 3), meaning these "mm-sized" grains would produce too much infrared radiation before they create $880 \mu \mathrm{m}$ residuals (when $M_{\text {cav }} \lesssim 10^{-8} \mathrm{M}_{\odot}$ ). Nevertheless, the example is instructive in pointing out that the $880 \mu \mathrm{m}$ data are not quite sensitive enough to provide very strong limits on $\delta_{\text {cav }}$. 

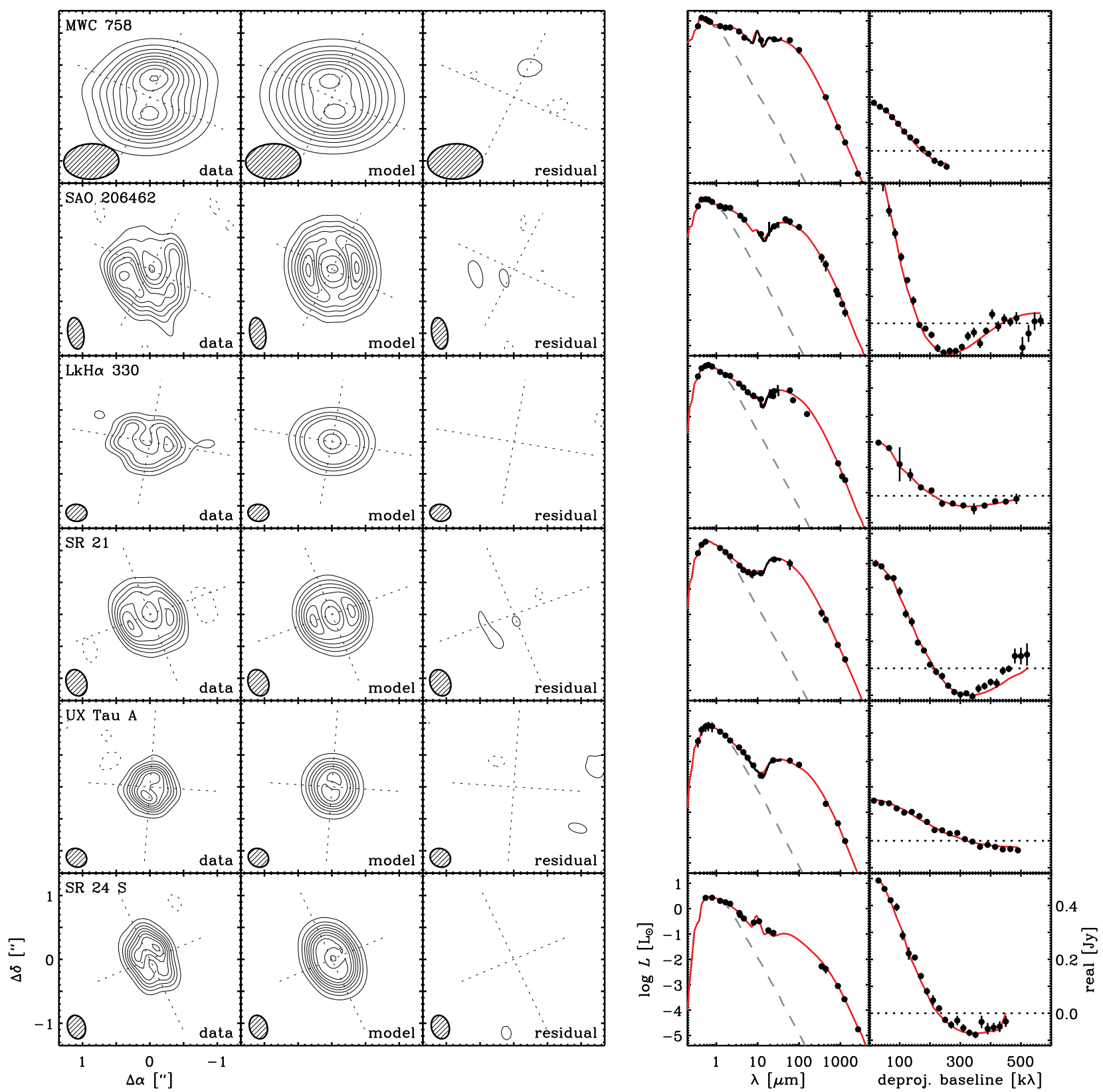

Figure 6. Comparisons of the disk structure models with observations. The left panels show the SMA continuum image, the model, and the imaged residuals, with $3 \sigma$ contour intervals (see Table 21). Crosshairs mark the disk center (see $\S 2$ and Table 1) and major axis orientation; their relative lengths represent the disk inclination. The right panels show the SEDs and $880 \mu \mathrm{m}$ visibility profiles, with models overlaid in red. The stellar photosphere models are marked with dashed curves.

$135 \mathrm{AU}$ and $M_{d}=0.006-0.128 \mathrm{M}_{\odot}$ inferred here (assuming a 100:1 gas-to-dust mass ratio). Surprisingly, the transition disks are distributed along the same $M_{d^{-}} R_{c}$ correlation that was noted for normal disks in Ophiuchus (Andrews et al. 2010b), with essentially the same scatter. Of course, disk mass comparisons between these two populations are misleading, as the cavities in transition disks are substantial mass reservoirs in normal disks. The same disk models without dust-depleted cavities (i.e., if $\Sigma=\Sigma_{g}$ at all radii) would be $\sim 1.3-3 \times$ more massive than indicated in Table $4(\sim 20 \times$ for the disk with the largest cavity, MWC 758). This implies that the surface densities in the outer disk are typically up to a few times higher in transition disks compared to their normal disk counterparts.
With a relatively small and potentially heavily-biased sample, there is little that can be concluded from any trends between the model structure parameters. As noted above, the outer parts of the transition disks do seem to follow the tentative $M_{d}-R_{c}$ correlation identified from a sample of normal $\mathrm{T}$ Tauri disks Andrews et al. 2010b). With a brief examination of the figures here (with the disks ordered by spectral classification), one might notice a possible trend between the stellar type and cavity size. Indeed, there is a marginal hint of a positive correlation $(\sim 2.5 \sigma)$ between $R_{\text {cav }}$ and $T_{\text {eff }}$ (also $L_{*}$ and $M_{*}$ ), such that the earlier type stars appear to have disks with larger cavities. However, the trend is likely an artifact of the endpoints being outliers: the low-mass stars host disks with small cavities (DM Tau, WSB 60), 

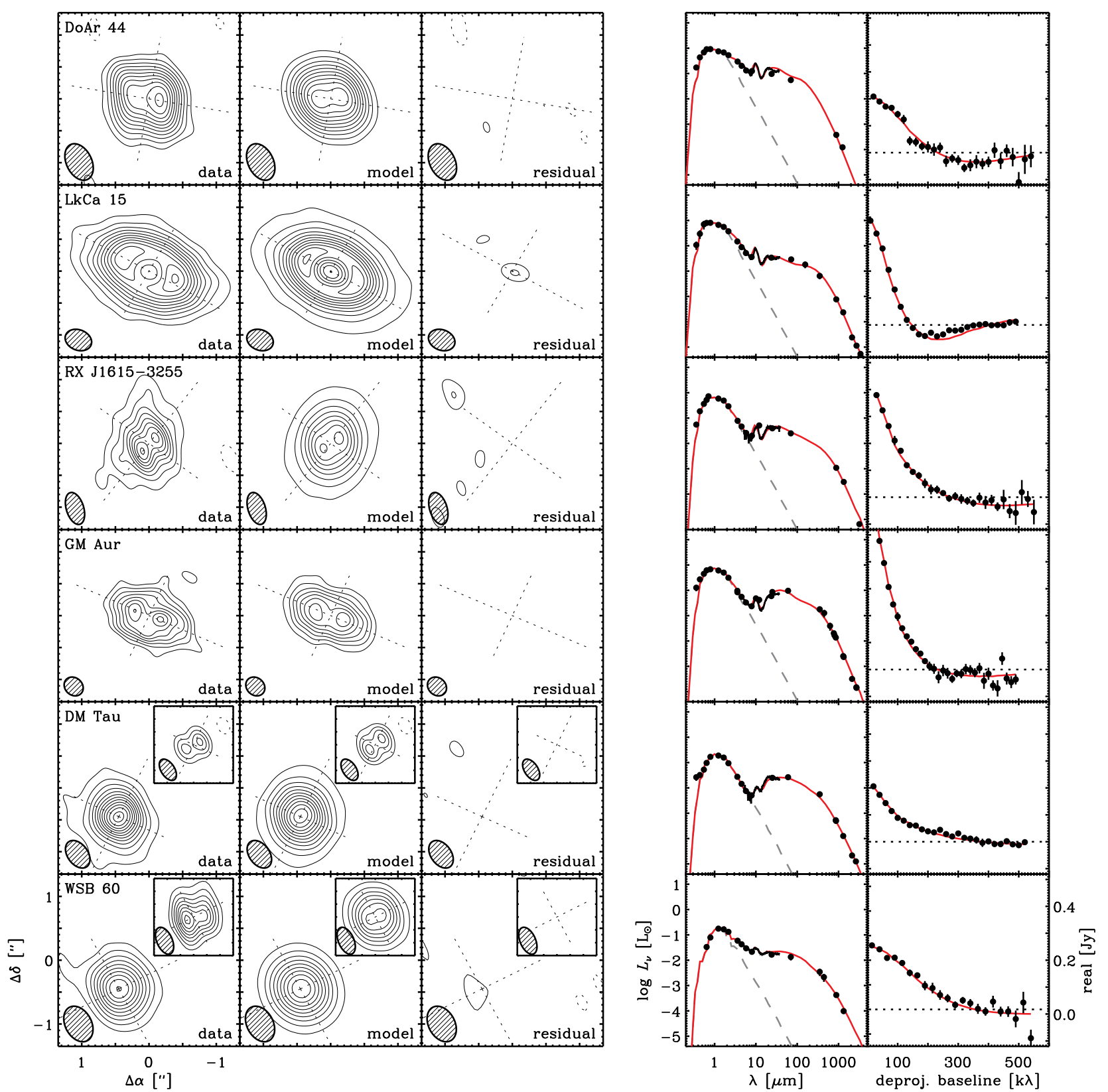

Figure 7. Same as Figure 6 The images for DM Tau and WSB 60 are slightly offset to make room for the inset maps that were synthesized with higher angular resolution (see Table 2).

and some of the higher-mass stars have disks with larger ones (MWC $758, \mathrm{LkH} \alpha 330$ ), but there is a wide range of $R_{\text {cav }}$ for intermediate cases. No other trends among the disk model parameters, or between them and the stellar properties, are found here. A more individualized description of the model results for each disk, along with a brief comparison to previous models when available, can be found in the Appendix.

\section{DISCUSSION}

\subsection{Transition Disks and Evolution}

5.1.1. Nomenclature and Our Definition of Transition Disk

Before integrating this sample of transition disks into a larger evolutionary context, it seems necessary to comment on its placement in the rather disorienting landscape of "evolved" disks in the current literature. Some of that confusion is related to nomenclature, and some is due to the inherently subjective nature of empirical classification. Our definition of a "transition" disk is conservative: we mean only those cases with unequivocal evidence for a sharp drop in the dust optical depths inside some radius, either in the form of a dip and then dramatic rise in the infrared spectrum over a narrow wavelength interval and/or a clearly resolved cavity at the disk center. Various other types of disks have been associated with the transition phase, including cases without a distinctive dip in the infrared. Such disks - sometimes termed "anemic" or "homologouslydepleted" - have relatively faint infrared excesses and often low accretion rates, but do not (yet) exhibit any compelling observational evidence for a dust-depleted cavity (Lada et al. 2006; Najita et al. 2007; Cieza et al. 


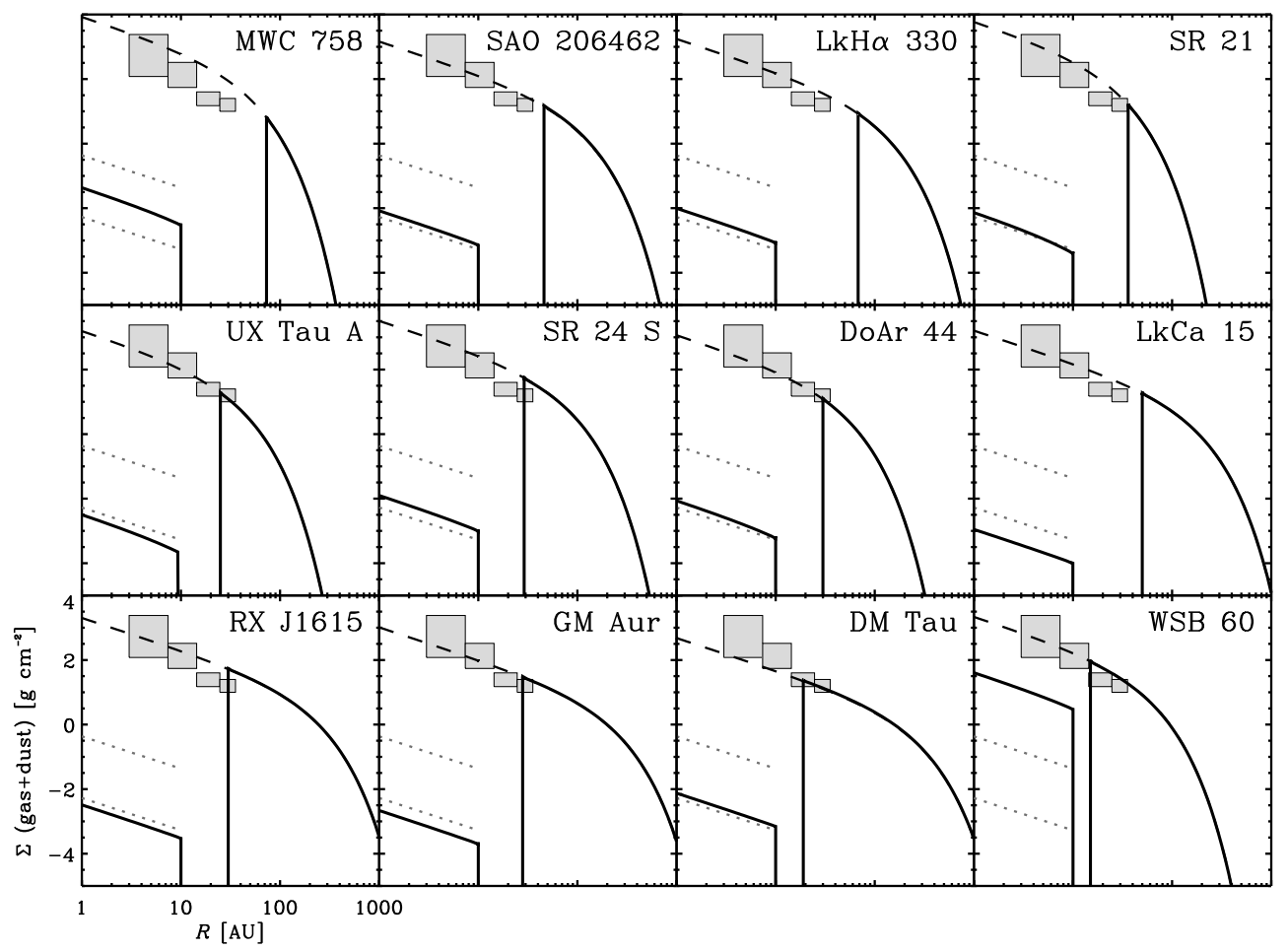

Figure 8. Model surface density profiles (solid curves), assuming a gas-to-dust mass ratio of 100:1. The underlying global density profiles, $\Sigma_{g}$ (see Eq. 1) are overlaid as dashed curves. The $\Sigma$ gradient parameter was fixed in the modeling to $\gamma=1$, meaning that $\Sigma \propto 1 / R$ at small radii $\left(R<R_{c}\right)$ and $\Sigma \propto 1 / e^{R}$ at large radii $\left(R>R_{c}\right)$. Intended as references, the gray squares correspond to the estimated surface densities in the Minimum Mass Solar Nebula (MMSN), a representation of the primordial, solar-composition disk densities required to account for the current giant planet masses in our solar system (and their uncertainties and feeding zones; Weidenschilling 1977b). Dotted lines in the inner disk mark the expected surface densities for 1 earth mass and 1 lunar mass of material (gas + dust), assuming $\Sigma \propto 1 / R$ from 0.1-10 AU. Note that the material inside the disk cavities is not resolved with our SMA observations; the projected resolution limits correspond to $R \approx 20-40 \mathrm{AU}$ (depending on the distance to an individual system).

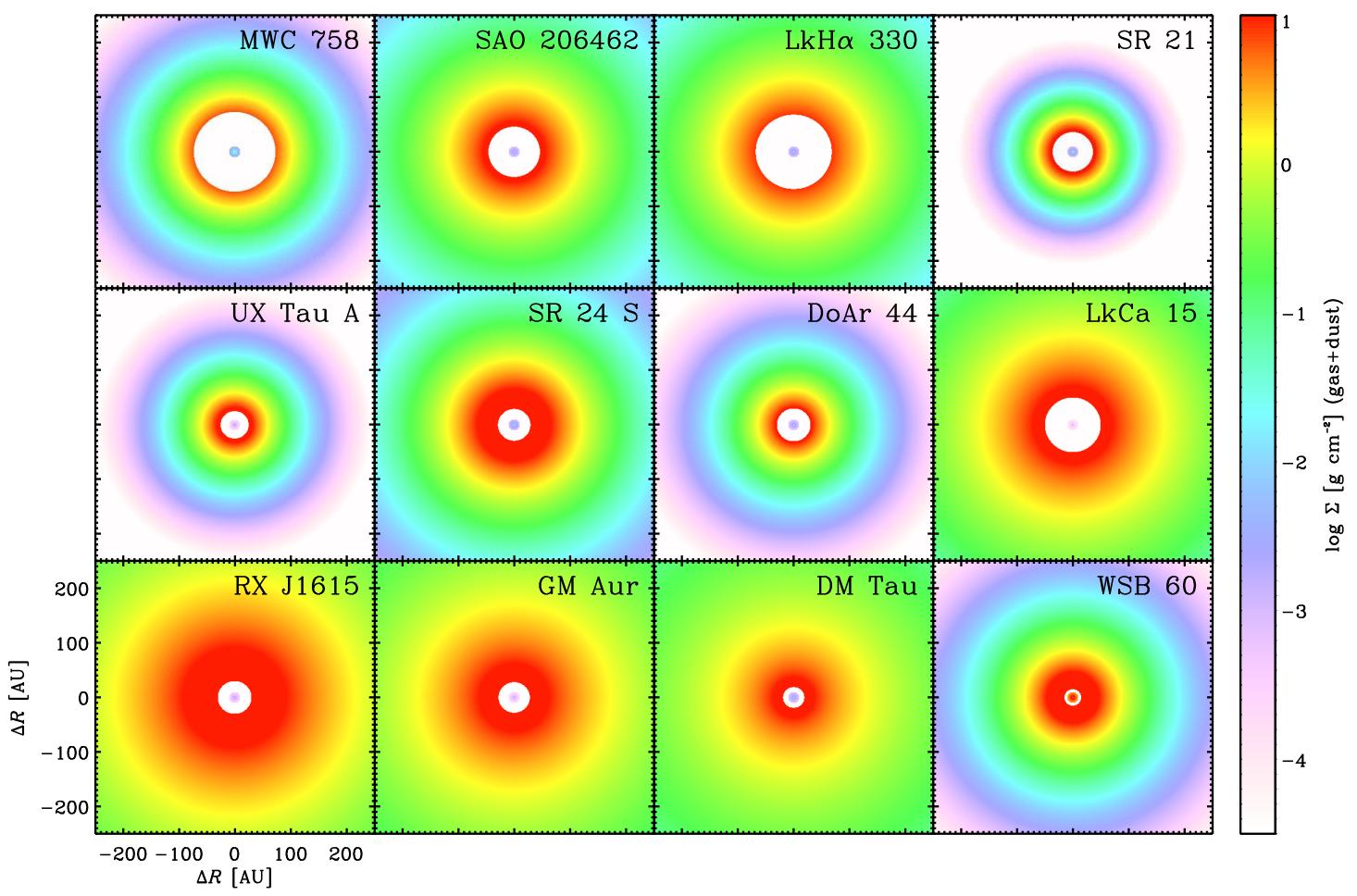

Figure 9. The model surface densities in a map format are shown together with the same color stretch (a $\Sigma$ scale-bar is on the right). This linear representation of the $\Sigma$ profiles provides a clear illustration of the range of cavity sizes, outer disk densities, and sizes $\left(R_{c}\right)$. 
2008, 2010; Currie et al. 2009; Currie \& Kenyon 2009). While they may signal an interesting evolution phase in their own right, these anemic disks are not bona fide transition disks in the sense intended here. Instead, the transition disks (in our adopted meaning) are typically identified with a set of specialized infrared color criteria (Cieza et al. 2007, 2010; Fang et al. 2009; Muzerolle et al.|2010; Merín et al.|2010). However, none of these color-selection methods successfully recovers our entire sample (with failure rates up to $60 \%$ ), despite the unambiguous confirmations of large disk cavities from the SMA (the Merín et al. "cold disk" criteria perform well; a slight expansion of their selection-space would encompass nearly all the disks in this sample).

These low recovery rates are not surprising, especially given the sometimes bright (and complex) emission spectrum generated by a small amount of dust inside the disk cavities. Due to the bewildering diversity of possible infrared SED behaviors for transition disks, any (unresolved) infrared-based identification scheme is biased and will miss good candidates. Unfortunately, our current ignorance of the material and structural properties of the inner disk makes it difficult to properly characterize those biases. In the end, the only appropriate systematic way to find and characterize the transition disk population is with a flux- and resolution-limited survey that can directly image dust-depleted cavities. Since that type of unbiased survey is not yet feasible, we focus on the small piece of selection-space that is (at least partially) addressed by the specific type of transition disks in this sample: namely, those with bright millimeter emission and large cavities.

\subsubsection{Frequency of Transition Disks with Large Cavities}

Despite the narrowed scope, transition disks with large cavities are surprisingly common when viewed in the proper context. Consider the millimeter-wave luminosities from disks in the nearby Tau and Oph associations (Andrews \& Williams 2005b, 2007a; Cieza et al. 2010). Removing non-detections, sources with likely envelope contamination (including flat-spectrum objects), and disks around early-type (A/B) stars, and scaling $1.3 \mathrm{~mm}$ photometry when necessary by a conservative factor $1 / \lambda^{3}$ (steeper than the typical slope found by Andrews \& Williams), we constructed the distribution of $880 \mu \mathrm{m}$ luminosities $\left(L_{\mathrm{mm}}\right)$ shown in Figure 10 for 91 disks. Out of that sample, large cavities $\left(R_{\text {cav }} \gtrsim 15 \mathrm{AU}\right)$ have been directly resolved for 9 disks (10\%, including RY Tau; see Isella et al. 2010a). That fraction is roughly consistent with expectations from infraredselection methods (Muzerolle et al. 2010), especially if the upper limits for millimeter-faint disks are included. But current disk imaging surveys are sensitivity-limited and have only probed the brightest disks. In the upper half of the $L_{\mathrm{mm}}$ distribution $(\geq 80 \mathrm{mJy}$ at $140 \mathrm{pc}$ ), the transition disks with large cavities comprise a larger fraction, $\sim 20 \%$ (9/46 disks). And in the upper quartile of the distribution ( $\geq 200 \mathrm{mJy}$ at $140 \mathrm{pc}$ ), that fraction is slightly higher still (26\%; 6/23 disks). These transition disk fractions are lower bounds; there could very well be more disks with large cavities that have yet to be imaged at sufficient resolution. The maximum fraction of this type of transition disk in the upper half (or quartile) of the $L_{\mathrm{mm}}$ distribution is $\sim 70 \%$, based on the known

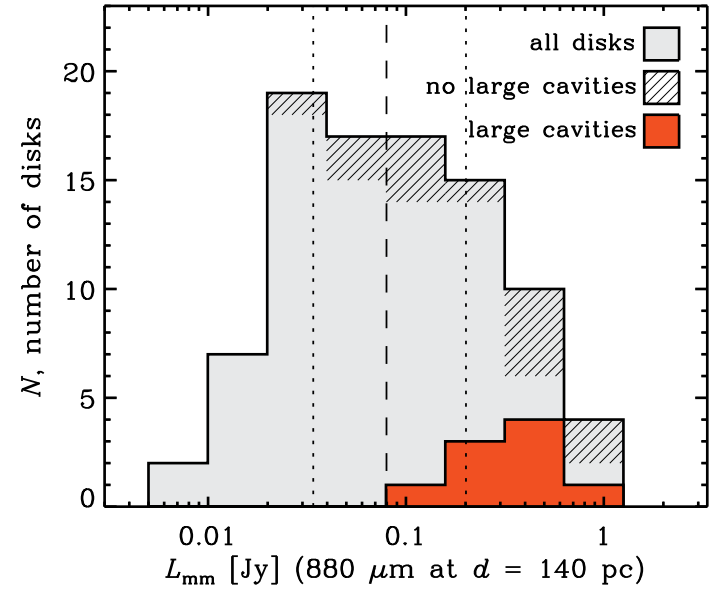

Figure 10. The distribution of $880 \mu \mathrm{m}$ luminosities $\left(L_{\mathrm{mm}}\right)$, scaled to a distance of $140 \mathrm{pc}$, for disks in the Taurus and Ophiuchus starforming regions (grey), excluding non-detections, sources with potential envelope contanimation (including flat-spectrum objects), and early-type (A/B) stars (data from Andrews \& Williams 2005b, $2007 \mathrm{~b})$. The median and quartiles of the distribution are shown as dashed and dotted lines, respectively. The $L_{\mathrm{mm}}$ distribution of the subsample of Tau/Oph millimeter-bright transition disks with directly resolved large $\left(R_{\text {cav }} \gtrsim 15 \mathrm{AU}\right)$ cavities is shown in red. The hatched regions correspond to disks that do not have large cavities, as determined from millimeter-wave observations with comparable resolution to this study (Andrews et al. 2009, 2010b). In each bin, the fraction of transition disks with large cavities is at least $N($ red $) / N$ (grey) and at most $N$ (hatched) $/ N$ (grey). That fraction is at least $20 \%$ (and at most $70 \%$ ) for the upper half of the $L_{\mathrm{mm}}$ distribution, and at least $26 \%$ (at most $70 \%$ ) for the upper quartile. As indicated by the large number of disks that lie in the unhatched grey areas of the $L_{\mathrm{mm}}$ distribution, many disks have not yet been observed at sufficient resolution to detect a dust-depleted cavity. Moreover, we do not yet know the transition disk fraction at low $L_{\mathrm{mm}}$ (below the median) or smaller $R_{\text {cav }}$.

absence of large cavities in some of the remaining disks (Andrews et al. 2009, 2010b).

To reiterate those remarkable statistics: at least 1 in 5, and possibly up to $\sim$ two-thirds of the known millimeter-bright disks have large dust-depleted cavities (with $R_{\text {cav }} \gtrsim 15 \mathrm{AU}$ ). We do not know how high such fractions might be for smaller cavities, or disks with even fainter millimeter emission, or young disk populations in other environments (if these results for Tau/Oph are to be applied to other clusters, one must keep in mind the counting uncertainties that correspond to $\pm 10 \%$ errors on the transition disk fractions quoted above). Regardless, the implication is that a large fraction of the most millimeter-bright - and therefore most massive - disks show unequivocal evidence for substantial dust evolution in the regions most relevant for planet formation on timescales of less than $\sim 1-2 \mathrm{Myr}$ (for reference, the median and quartile $L_{\mathrm{mm}}$ values correspond to disk masses of $0.005 \mathrm{M}_{\odot}$ and $0.011 \mathrm{M}_{\odot}$, respectively, using the standard Andrews \& Williams assumptions). This reinforces a similar conjecture made by Najita et al. (2007) based on a group of unresolved Taurus disks, although see $\S 5.3 .3$ regarding their corollary claim of low accretion rates.

\subsubsection{Comments on Evolution}

In retrospect, it is striking that these transition disks could be such bright millimeter sources in spite of their depleted cavities. What were these disks like before they evolved, and are such progenitor disks still present in 
these young associations? If we assume that these disks had $\Sigma=\Sigma_{g}$ at all radii before depleting their cavities, our radiative transfer calculations indicate that $L_{\mathrm{mm}}$ would typically be $\leq 2 \times$ larger (up to $4 \times$ for the extreme case, MWC 758). Note that the emission increase is not directly proportional to the mass increase expected from filling the cavity with dust (see $\S 4$ ), due to optical depth effects. Unfortunately, there are only a few such disks on the extreme tail of the $L_{\mathrm{mm}}$ distribution. With such low numbers, identifying a parent population for these massive transition disks is difficult. It is possible that they represent the evolved versions of massive flat-spectrum sources (e.g., DG Tau, HL Tau). Or perhaps some of them brightened after depleting their cavities: for example, viscous spreading of an initially narrow optically thick ring could increase $L_{\mathrm{mm}}$ (if the flux scales with the disk size, $R_{c}$; see Andrews et al. 2010b).

The cavity sizes and millimeter luminosities are not the only clues available to help characterize transition disk evolution, and we would be remiss not to address potential hints from the cavity contents. In terms of their near-infrared excess luminosities, it is clear that not all transition disks are alike: some are faint (in this sample, DM Tau, GM Aur, RX J1615-3255, and perhaps SR 21) and others are quite bright (e.g., DoAr 44). Espaillat et al. (2007) suggested that the latter cases, dubbed "pre-transition" disks, may be more representative of gaps than cavities. That hypothesis (and accompanying nomenclature) supposes that the transition disks with brighter infrared excesses trace an earlier stage of the inner disk depletion process. That may very well be the case, but the properties of the inner disk are so poorly constrained that it is not the only possibility. While there is no clear association of infrared excess luminosity with the depletion factor, $\delta_{\text {cav }}$, we were forced to remove dust inside $\sim 1$ AU to avoid over-producing the observed emission for our DM Tau and RX J1615-3255 models (see the Appendix). That is in good agreement with the original Espaillat et al. (2007) conjecture, where the inner disk densities (or optical depths) and excess levels are related. However, the strength of the infrared excess is also tied to the vertical distribution of dust near the star: larger heights generate more emission (see $\S 3$, or Espaillat et al. 2010). Perhaps the transition disks with brighter infrared emission also have more "puffed"-up inner rims. This might suggest that those cases with faint excesses have relatively lower inner disk gas densities (or gas-to-dust ratios), which do not provide sufficient pressure to support such extended vertical structures.

\subsection{Potential Dust Depletion Mechanisms}

Up to this point, the discussion has been intentionally generic with respect to the physical origins of the dustdepleted cavities in transition disks. In a broad sense, there are two types of depletion mechanisms: those that physically remove or "clear" material from the inner disk (decrease $\Sigma$ ), and those that modify the emission properties of the dust (decrease $\kappa$ ). Various inner disk evolutionary processes have been assessed in previous studies of transition disks (D'Alessio et al. 2005; Najita et al. 2007; Alexander \& Armitage 2009). We review several of the proposed dust depletion mechanisms in the context of the specific type of transition disk in our sample.

\subsubsection{Dispersal by Winds}

Among the proposed clearing mechanisms, mass-loss in a photoevaporative wind has excellent potential for clearing a disk cavity Clarke et al. 2001; Alexander et al. $2006 \mathrm{a}, \mathrm{b})$. Energetic photons from the central star can ionize the disk surface layers and launch a wind from just inside the radius where the thermal energy of the ionized gas exceeds the escape velocity $(R \approx 1-10 \mathrm{AU}$; Shu et al. 1993; Hollenbach et al. 1994). If the inward mass (accretion) flow rate exceeds the mass-loss rate in this wind, changes to the disk structure occur slowly, as expected for viscous evolution. But as the accretion flow decays over time, it will eventually be surpassed by the mass-loss rate in the wind (assuming the photoionizing flux from the star remains high; Alexander et al. 2004, 2005). At that point, the accretion flow is redirected into the wind and gas is rapidly depleted in a gap near the wind launch radius. Isolated from its replenishment source (the outer disk), the inner disk material quickly drains onto the star. The initially small cavity made in this way exposes the disk edge near the wind launch radius to unattenuated high-energy irradiation, accelerating the dispersal of material and growth of the cavity. Although this photoevaporation process only directly affects the gas phase of the disk (only the very smallest dust grains, $s \leq 0.1 \mu \mathrm{m}$, are directly swept away in the wind), Alexander \& Armitage (2007) demonstrated that a simple prescription for the radial drift of solids promotes the simultaneous depletion of dust inside the wind-blown cavity.

In models that consider only UV irradiation, the wind plays a minor role in the evolution of a disk structure over most of its lifetime (Alexander et al. 2006b; Gorti et al. 2009). Only late in life, when the disk mass and accretion flow have diminished, does photoevaporation dramatically influence the disk properties. Observationally, the transition disks produced by UV photoevaporation should have empty cavities, low accretion rates $\left(\dot{M} \leq 10^{-10} \mathrm{M}_{\odot} \mathrm{yr}^{-1}\right)$ and low masses $\left(M_{d} \leq 0.005 \mathrm{M}_{\odot}\right)$, and orbit relatively "old" stars $(\sim$ few Myr; Alexander \& Armitage 2007, 2009). Given the large disk masses (Table 4) and high accretion rates (Table 3) in this sample, it is clear that UV photoevaporation is not responsible for creating the large cavities in these millimeter-bright transition disks (as noted by Alexander \& Armitage 2007). However, there is growing consensus that X-rays drive substantially higher wind mass-loss rates, and therefore that photoevaporation has a much more prominent effect on disk structures at early times (Ercolano et al. 2008, 2009; Drake et al. 2009; Owen et al. 2010). The early onset of X-ray photoevaporation implies that these disks could still have material in their cavities, and may have higher accretion rates (up to $\left.10^{-8} \mathrm{M}_{\odot} \mathrm{yr}^{-1}\right)$ and disk masses $\left(M_{d} \sim\right.$ $0.01 \mathrm{M}_{\odot} ;$ Owen et al. 2011). To assess the role of X-ray photoevaporation, we compare the quiescent X-ray luminosities ( $L_{X}$ in Table 3) with the cavity sizes $\left(R_{\text {cav }}\right)$, disk masses, and accretion rates. In this sample, the $R_{\text {cav }}$ and $\dot{M}$ values for a given $L_{X}$ are much too large to be consistent with their formation via photoevaporative mass-loss (as noted by Owen et al. 2011). That is not to say that photoevaporation is unable to clear disk cavities, as it seems a likely explanation for some evolved disks (e.g., Cieza et al. 2008; Pascucci \& Sterzik 2009) - 
only that it is insufficient to explain the population of massive transition disks with large cavities.

Some alternative wind sources have been suggested as potential contributors to the clearing of disk material. Based on their local MHD simulations of turbulence driven by the magneto-rotational instability (MRI), Suzuki \& Inutsuka (2009) postulated that the disruption of large-scale channel flows by magnetic reconnection events can drive a substantial wind off the disk surface. Extrapolated to global evolution models for the disk structure, the mass-loss rates in the inner disk from such a wind could rival those produced by X-ray photoevaporation (Suzuki et al. 2010). Those initial calculations suggest that $R_{\text {cav }} \lesssim 15 \mathrm{AU}$ cavities could be depleted on Myr timescales, but a more comprehensive set of observational predictions that can be used to test the feasibility of MRI disk winds as a transition disk clearing mechanism (and differentiate it from photoevaporation) is not yet available. Aside from winds launched from the disk itself, the wind generated by the central star may similarly facilitate disk dispersal by stripping material out of the disk atmosphere (e.g., Horedt 1978). Recent models by Matsuyama et al. (2009) indicate that the mass-loss rate of disk material from this process is quite small for young disks, although perhaps the stellar wind can act in concert with disk winds to accelerate the dispersal of the inner disk. That said, the potential synergy of these various wind dissipation models has not yet been explored in detail.

\subsubsection{Material Evolution - Particle Growth}

Instead of a lack of mass in the inner disk, the cavities we observe could instead be a sign that substantial material evolution has occurred in the inner parts of transition disks. Solid particle growth is a natural disk process that might be able to explain the transition disk structures without requiring drastic density modifications. Because large solid particles have low opacities $(\kappa)$, significant grain growth can diminish the continuum emission over a wide wavelength interval (Mivake \& Nakagawa 1993; D'Alessio et al. 2006). To produce the observed infrared SED deficits with a continuous density profile $\left(\Sigma=\Sigma_{g}\right)$, the $\sim 1-10 \mu \mathrm{m}$ opacities in the inner disk need to be decreased by a factor of roughly $\delta_{\text {cav }}$ (because the infrared emission sets the contrast parameter; see §3.5). The $880 \mu \mathrm{m}$ opacity also needs to decrease by a factor of $\geq 10$ 100 , to account for the ring-like emission observed with the SMA. With the dust composition assumed here, such opacity spectra are only possible after growth beyond $s_{\max }=1 \mathrm{~m}$ (preferably with a top-heavy size distribution, $p \leq 2.5)$. Coagulation timescales are expected to be shorter in the inner disk, due to the increased densities and relative velocities closer to the central star. In principle, that would produce a radial variation in particle sizes and a decrease in $\kappa$ - and therefore emission toward smaller disk radii.

Nevertheless, there are challenges to reproducing the transition disk cavities with particle growth alone. For example, models of the grain size evolution in disks predict smooth $\kappa$ variations (Dullemond \& Dominik 2005; Garaud 2007; Brauer et al. 2008; Birnstiel et al. 2010), rather than the abrupt optical depth change near $R_{\text {cav }}$ that is observed. To account for the sharp optical depth contrast signaled by the $880 \mu \mathrm{m}$ emission rings (i.e., the visibility nulls) and the shape of the mid-infrared spectrum, particle sizes would need to increase dramatically over a narrow radial range (at most a few AU). That type of break in $\kappa(R)$ might be reproduced if particle growth is significantly enhanced in a large, quiescent dead zone with a sharp outer edge. In a dense disk, the midplane can be so shielded from ionizing radiation that the MRI will not operate (Gammie 1996; Sano et al. 2000; Fromang et al. 2002): without that source of disruptive turbulence, particle growth can proceed more efficiently inside the dead zone. But there are two problems with this scenario. First, the disk atmosphere will remain partially ionized and can channel material from the outer disk toward the star in surface accretion flows (e.g., Gammie 1996). Even small amounts of $\mu \mathrm{m}$-sized dust grains carried along in that flow could produce enough infrared radiation to wash out the distinctive dip in a transition disk SED. And second, the cavities are much larger than is expected for dead zones (Matsumura \& Pudritz 2003). Even in a large dead zone without dust in the surface layers, a collisional fragmentation process might still produce a substantial reservoir of small grains and limit growth beyond $\sim$ cm-scales (Brauer et al. 2008; Birnstiel et al. 2010, 2011). New models of particle growth under dead zone conditions (with radiative transfer) are needed to determine if coagulation and fragmentation in a low-turbulence environment can produce an infrared spectrum and $880 \mu \mathrm{m}$ emission ring consistent with those observed for transition disks.

Molecular emission tracers can provide additional insight here, as particle growth itself is not expected to significantly perturb the gas $\left(\mathrm{H}_{2}\right)$ densities inside the cavity. The measured accretion rates (Table 3 , aside from SR 21) unambiguously confirm that neutral gas is flowing through these cavities and onto the stars. Also, some of these transition disks emit $\mathrm{CO}$ rovibrational spectra that attest to the presence of warm molecular gas in their inner few AU (Najita et al. 2003; Salvk et al. 2007, 2009; Pontoppidan et al. 2008). However, new studies indicate that those inner regions are deficient in other molecules (e.g., $\mathrm{H}_{2} \mathrm{O}$; see Naiita et al. 2010; Pontoppidan et al. 2010), and Dutrey et al. (2008) claim that the GM Aur disk has a $R \approx 20$ AU region of depleted $\mathrm{CO}$ emission. In some respects, it is clear that transition disks have different molecular signatures compared to their normal disk counterparts. But given the inherent challenges in interpreting such measurements, it remains uncertain whether their cavities also have depleted gas densities (other effects like photodissociation, selfshielding, and gas-grain chemistry must also be considered; e.g., Aikawa \& Nomura 2006; Jonkheid et al. 2007; Vasvunin et al. 2011). Nevertheless, we can expect that these molecular emission lines will be crucial in evaluating what role particle growth has in producing the observed dust structures of transition disks.

\subsubsection{Tidal Interactions with Companions}

A large fraction of stars have companions Abt \& Levy 1976; Duquennoy \& Mayor 1991), with stellar multiplicity fractions approaching $75 \%$ in the diffuse young clusters near the Sun (e.g., White \& Ghez 2001; Kraus et al. 2011). Circumstellar material is expected to be strongly affected by dynamical interactions with these stellar com- 
ponents (see Lin \& Papaloizou 1993). The interplay of resonant and viscous torques can open a gap in a circumsystem disk, truncate individual disk edges, and potentially expedite disk dissipation (via accretion or ejection), depending on the orbital separation $(a)$, eccentricity $(e)$, and mass ratio $(q)$ of the companion. Based on the calculations of Artymowicz \& Lubow (1994), a binary with a large separation can retain individual disks similar to those around single stars, but with their outer edges truncated at $R_{O} \sim 0.2-0.5 a$. For smaller separations, a circumbinary ring structure may also be present, with a truncated inner edge at $R_{c b} \sim 1.8-2.6 a$. Pichardo et al. (2008) find similar results, but generalize to arbitrary eccentricities such that $R_{c b} \sim 1.9\left(1+e^{0.3}\right) a$. Their calculations suggest that the inner radius of the circumbinary ring has only a weak dependence on the mass ratio, at least for $q \geq 0.1$. It is natural to associate the $880 \mu \mathrm{m}$ emission rings observed for the transition disks with these circumbinary structures. In fact, stellar companions have been detected inside the cavities inferred (indirectly) for a few notable transition disks, including CoKu Tau/4 (Ireland \& Kraus 2008) and the older HD 98800 and Hen 3-600 systems (Uchida et al. 2004; Furlan et al. 2007; Andrews et al. 2010a).

If the transition disk cavities were created by tidal interactions with stellar companions, their expected orbital separations would be $a \sim 0.3-0.6 R_{\text {cav }}$, for high eccentricities $(e \sim 0.9)$ down to circular orbits. The range of cavity sizes measured here would correspond to companion separations of $a \sim 4-40$ AU. The multiplicity fraction for similar primary star masses in this separation range is 10-20\% (Duquennov \& Mavor 1991; Kraus et al. 2011), which is similar to, but slightly below, the minimum frequency of millimeter-bright transition disks with large cavities we estimated in $\S 5.1 .2$. Given how small those separations are projected on the sky, $\sim 0.04-0.20^{\prime \prime}$, direct searches for companions have been limited by both resolution and contrast. Kraus et al. (2011) have overcome such restrictions in Taurus, using a near-infrared aperture-masking interferometry technique coupled with the adaptive optics system on the Keck telescope. For the four Taurus disks in our sample (as well as RY Tau; see Isella et al. 2010a), Kraus et al. definitively rule out the presence of any companions with $q \geq 0.01$ ( $\geq 0.02$ for DM Tau) in the separation ranges implied by $\bar{R}_{\text {cav }}$ (see also Pott et al. 2010). The same applies to the $\mathrm{LkH} \alpha 330$ and SR 21 disks (A. L. Kraus 2011, private communication). For at least half of the known transition disks with large cavities, there are no companions with $M_{s} \gtrsim 20$ $30 \mathrm{M}_{\text {jup }}$ that could be responsible for the observed disk structures.

Until similar measurements are available for the other transition disks, their multiplicity status will remain uncertain. However, there are some stark differences between the transition disks and $a=4-40$ AU multiples. Tidal interactions substantially diminish disk masses in multiples with this separation range (Jensen et al. 1994, 1996; Osterloh \& Beckwith 1995; Andrews \& Williams 2005b; Cieza et al. 2009). To illustrate that point, we compare the millimeter-wave luminosities $\left(L_{\mathrm{mm}}\right.$, see $\S 5.1 .2)$ with the projected separations of the known multiples in Ophiuchus and Taurus (Barsony et al. 2003; Kraus et al. 2011) in Figure 11. The transition disks are marked with red lines, representing the companion separation ranges implied by their cavity sizes. More directly, Figure 11] compares the cumulative $L_{\mathrm{mm}}$ distributions of the transition disks and the known 4-40 AU multiples (the latter includes upper limits and was constructed using the Kaplan-Meier product limit estimator; see Feigelson \& Nelson 1985). The two populations have remarkably different $L_{\mathrm{mm}}$ (or equivalently, $M_{d}$ ) distributions: the median transition disk is at least $30 \times$ brighter (more massive) than the 4-40 AU multiples. Of the 44 known multiples in that separation range, only one - GG Tau - is brighter than $30 \mathrm{mJy}$ at $880 \mu \mathrm{m}$. GG Tau is exceptionally rare, hosting a circumbinary ring not unlike the transition disk structures (it technically meets our definition of a transition disk in \$5.1.1), but with an enormous $R_{c b} \approx 180 \mathrm{AU}$ (Guilloteau et al. 1999). In any case, the absence of millimeter-bright 4-40 AU multiples is striking: it suggests that the specific type of transition disk studied here has not suffered from the same destructive interaction mechanism.

Some caution is warranted in over-interpreting the differences between these two populations, as selection biases, unknown orbital parameters $(e, i)$, and perhaps uncertainties in the tidal truncation models (e.g., see Beust \& Dutrev 2005) should also be considered. One notable distinction is related to the mass ratio. There are stringent limits on $q(\geq 0.01-0.02)$ for at least half of the transition disk sample, but the 4-40 AU multiples are preferentially equal-mass (median $q \approx 0.7$; Kraus et al. 2011). Such high mass ratios could impart more severe tidal perturbations to the circumstellar environment at an early evolutionary stage. Since that seems unlikely for the transition disks, it is worthwhile to explore the effects of companions with much lower $q$ values. Such low-mass companions that form in the disk around the primary - including giant planets and brown dwarfs - can modify the disk structure without dispersing too much of the total mass $\left(\propto L_{\mathrm{mm}}\right)$.

Resonant torques induced by a low-mass companion set up spiral waves that repel local disk material Goldreich \& Tremaine 1978, 1980; Lin \& Papaloizou 1979a , b). Above a mass ratio threshold, $q \gtrsim$ $q_{\text {gap }} \approx 40 \alpha(H / a)^{2}$ (where $\alpha$ is the viscosity coefficient; Shakura \& Sunyaev 1973), these tidal interactions open a gap in the disk (Lin \& Papaloizou 1986, 1993; Bryden et al. 1999; Crida et al. 2006). In this scenario, the companion is located closer to the outer edge of the cavity than for the high- $q$ case. A typical separation for a circular orbit might be $a \sim 0.8 R_{\text {cav }}$, although the exact location depends on some complicated issues that influence the gap width (Winters et al. 2003; Crida et al. 2006). For our model structures and $\alpha=0.01$, the transition disks in this sample would need companions with $a \sim 10-60 \mathrm{AU}$ and mass ratios at least $q_{\text {gap }} \approx 0.001-$ 0.03 to open gaps near their cavity edges. Using the $M_{*}$ values in Table [3, those limits translate to companion masses $M_{s} \gtrsim 1-70 \mathrm{M}_{\text {jup }}$ (the high end corresponding to larger $M_{*}$; note that $q$ gap and $M_{s}$ scale linearly with the assumed $\alpha$ ). For the relevant cases, the mass ratios implied from current infrared contrast limits (Pott et al. 2010; Kraus et al. 2011) exceed those needed to open gaps. Although reassuring, there is little to be learned there, given the uncertainties on the disk viscosities $(\alpha)$ and the luminosities of such low-mass companions (e.g., Marley et al. 2007). 

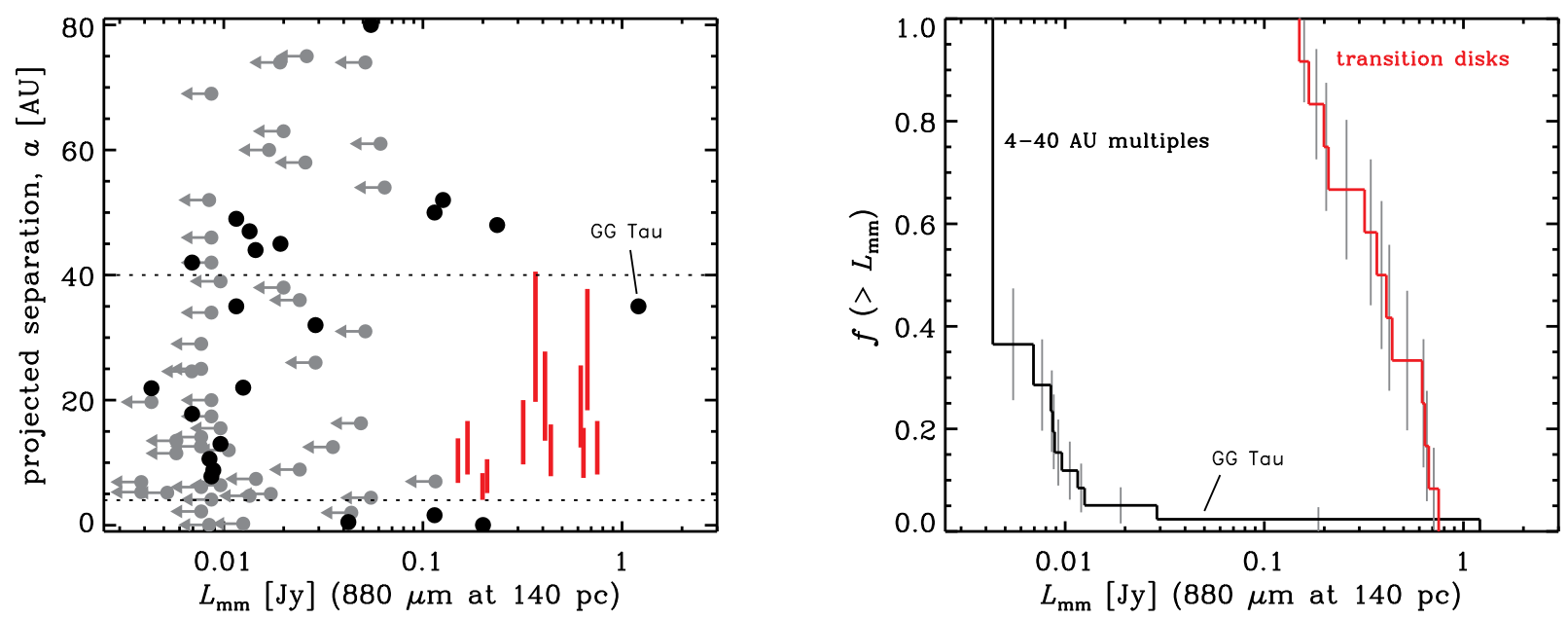

Figure 11. (left) The $880 \mu \mathrm{m}$ luminosity ( $L_{\mathrm{mm}}$; Andrews \& Williams 2005b, 2007b) as a function of the projected separation $(a$; Barsony et al. 2003; Kraus et al. 2011) for multiple star systems in the Taurus and Ophiuchus star-forming regions. Gray points with arrows signify $3 \sigma$ upper limits on $L_{\mathrm{mm}}$. The red lines mark the stellar companion separations that would be expected to clear the transition disk cavities: their vertical extents correspond to the range of eccentricities $e \sim 0.0-0.9$ Artvmowicz \& Lubow 1994; Pichardo et al. 2008). (right) A direct comparison of the cumulative $L_{\mathrm{mm}}$ distributions for the transition disks (red) and the multiples with projected separations of 4-40 AU (black; the latter was constructed with the Kaplan-Meier estimator to include $3 \sigma$ upper limits). The two populations have extremely different millimeter-wave luminosity (disk mass) distributions: transition disks are typically a factor of $\sim 30 \times$ brighter.

An individual gap is too narrow to be responsible for the observed properties of the transition disks. However, the disk-companion interactions can also regulate the densities in the inner disk, interior to the companion orbit. As in the case for a photoevaporative flow (§5.3.1), the gap effectively isolates the inner disk from its replenishment reservoir at larger radii. Without a viscous flow of material inwards, the inner disk material would drain onto the star on a relatively short timescale, producing a large and empty cavity. Unlike the photoevaporation mechanism, some material from the outer disk is expected to seep through a gap created by a low-mass companion in accretion streams, but only at decreased flow rates compared to a normal disk (e.g., Artymowicz \& Lubow 1996; Klev 1999; Lubow et al. 1999). Lubow \& D'Angelo (2006) estimate that the mass flow across such a gap is diminished by $\sim 75-90 \%$, depending on $q$ : a more massive companion reduces the flow across the gap, leading to a lowerdensity inner disk. A quantitative association between the companion mass, the accretion flow, and the depletion of the inner disk is not yet clear in simulations. Some have suggested a companion with $q \approx 0.001$ is sufficient to deplete a disk cavity like those observed for the transition disks (Rice et al. 2003a; Quillen et al. 2004; Varniére et al. 2006), while others have argued that higher masses are required (Crida \& Morbidelli 2007).

Najita et al. (2007) suggested that transition disks exhibit low accretion rates compared to their normal counterparts with similar disk masses, consistent with a large reduction in the mass flow across a gap produced by a low-mass companion (see also Alexander \& Armitage 2007, 2009). To verify that result, we constructed the distribution of $\dot{M}$ for normal disks in Taurus and Ophiuchus that lie in the bright half of the $L_{\mathrm{mm}}$ distribution and compared it with the accretion rates for the transition disks with large cavities in those regions (including RY Tau; see Table 31). Figure 12 shows the cumulative distributions of $\dot{M}$ for both of these populations. A twosided Kolmogorov-Smirnov test (or its survival analysis equivalents if we include the $\dot{M}$ upper limit for SR 21 ; see Isobe et al. 1986) indicates that the probability these $\dot{M}$ populations are drawn from the same parent distribution is low $(\leq 1-2 \%)$, with the transition disks having about $\sim 3 \times$ lower accretion rates on average. That relative $\dot{M}$ decrease ( $\sim 70 \%)$ is similar to the expected reduction in flow rates across a gap opened by a $q \approx 0.001$ (i.e., $M_{s} \approx 1 \mathrm{M}_{\text {jup }}$ ) companion (Lubow \& D'Angelo 2006). However, the samples are small, incomplete, and likely biased. Moreover, the individual accretion rates have large uncertainties and are estimated from different diagnostics. So, although this preliminary comparison (and that by Najita et al. 2007) provides a tantalizing hint of reduced mass flow rates for transition disks, we caution that a more rigorous and homogeneous set of comparisons is warranted before any conclusions are drawn.

Regardless of those comparisons, the $\dot{M}$ values for the transition disks are still high enough to be seemingly inconsistent with the low dust densities we infer inside their cavities. One way to reconcile those measurements is to include additional low-mass companions inside the observed disk cavity. Zhu et al. (2011) have suggested that multi-planet systems can dynamically clear large cavities while also rapidly transporting gas across the large distances between the remnant outer disk and the star. Alternatively, a single low-mass companion might be responsible for creating a cavity that is depleted in dust, but not in gas. Any companion that can open a gap will generate wakes just outside its orbit, creating a pressure bump that may act like a dust filter on the flow across the gap (Rice et al. 2006; Paardekooper \& Mellema 2006). If that filter removes a substantial fraction of the dust from the flow, the inner disk can be preferentially depleted of dust (producing the thermal deficits we observe) while still maintaining high gas accretion rates.

The tidal interactions between disk material and lowmass companions offer a compelling explanation for the transition disk structures. However, the observations are only starting to provide some tangible constraints that can be incorporated into the numerical simulations. As 


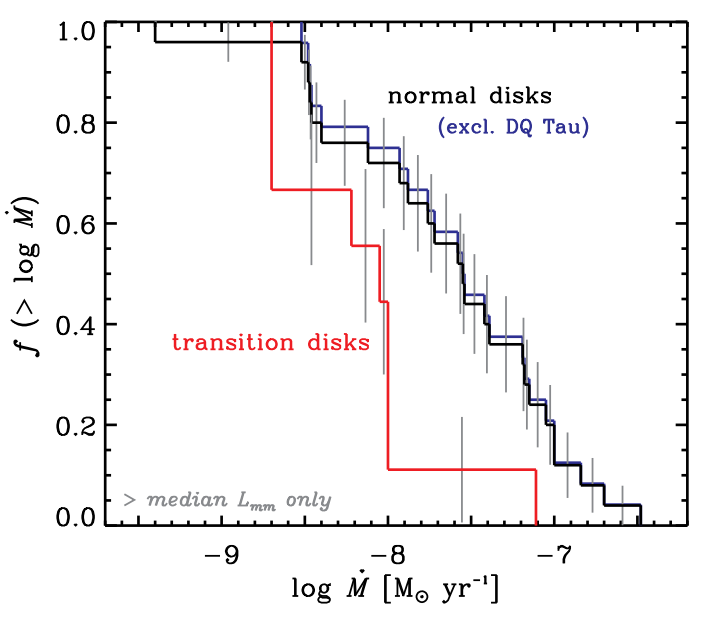

Figure 12. The cumulative distribution of the accretion rates $(\dot{M})$ for the normal disks in Tau and Oph that reside in the bright half of the $L_{\mathrm{mm}}$ distribution (black, or blue excluding the spectroscopic binary DQ Tau), compared with that for the transition disks with large cavities in those same regions (red; including RY Tau). The latter was constructed with the Kaplan-Meier estimator to include the upper limit on $\dot{M}$ for the SR 21 disk. We do not include the candidate transition disk RX J1633-2422 in either distribution, although it has a very low $M$ estimate (see Cieza et al. 2010). Accretion rates were compiled from Hartmann et al. (1998), Natta et al. (2006), and Naiita et al. (2007). Of the 46 millimeter-bright disks considered, 7 (primarily in Oph) have no $M$ measurements in the literature. In this preliminary comparison, the transition disks typically have accretion rates $\sim 3 \times(0.5 \mathrm{dex})$ lower than the normal disks. However, it is difficult to reach any definitive conclusions from such a comparison: the two samples are small and likely incomplete (and biased), and the $\dot{M}$ values have large uncertainties and were determined in different ways.

the data improve - both for the disk structures and companion searches - we should be able to make a more nuanced assessment of whether the mechanism responsible for transition disk cavities is tied directly to the formation and early evolution of low-mass brown dwarfs and/or exoplanets.

\subsection{Implications for Planet Formation}

To summarize the discussion in $§ 5.2$, we have argued that the dust-depleted cavities in the specific type of transition disks studied here - those with large $L_{\mathrm{mm}}$ and $R_{\text {cav }}$ - were not likely produced by photoevaporative winds or tidal truncation by stellar-mass companions. The possibility that the transition disk cavities are the manifestation of substantial particle growth in large, low-ionization dead zones remains, but requires a more detailed modeling effort to determine if some significant challenges could be mitigated. While these mechanisms all play non-trivial roles in disk evolution, the weight of the observational evidence for the kind of transition disks studied here suggests that the cavities are likely the byproduct of dynamical interactions with long-period low-mass companions - either brown dwarfs or giant planets. If that is the case, it is of great interest to understand how those companions can form at (or be moved to) such large separations from their host stars $(a \sim 10-60 \mathrm{AU})$ on such a relatively short timescale (at most $\sim 1-2 \mathrm{Myr}$ ).

Giant planets or low-mass brown dwarf companions could be formed relatively rapidly from a fragmentation process in a gravitationally unstable disk (e.g., Boss 1997, 1998; Rice et al. 2003b). Moreover, this disk instability mechanism seems to naturally favor formation at wide separations from the primary (Boss 2006; Bolev 2009; Rafikov 2009; Clarke 2009; Dodson-Robinson et al. 2009). Of course, it is not clear that the transition disks initially had sufficiently high densities (and efficient cooling) to be unstable, and in most cases (8/12 in this sample) the orbits of any companions would be small enough $(a \leq 35 \mathrm{AU})$ that the prospects for fragmentation are debatable (but see Boss 2009). If the companions are not too massive, the more standard core accretion mechanism for giant planet formation may be applicable. In that scenario, the collisional agglomeration of solids must produce a massive core to accrete a massive atmosphere before the gas disk disperses (e.g., Pollack et al. 1996; Hubickyj et al. 2005). Traditionally, that process has been considered too inefficient to make long-period planets $(a \gtrsim 10 \mathrm{AU})$ in situ within the disk lifetime. However, Rafikov (2011) has argued that an improved treatment of planetesimal accretion shows expedited core growth at low surface densities, thereby enabling the capture of a massive atmosphere in the outer disk. His estimate of the $\geq 0.1 \mathrm{~g} \mathrm{~cm}^{-2}$ of solids needed for that process is strikingly similar to (or below) the $\Sigma$ values we derive near $R_{\text {cav }}$ (see Figure 8). Others have suggested that long-period companions could be produced relatively easily by the core accretion mechnism in the inner disk, and then subsequently moved outward via planet-planet scattering (Scharf \& Menou 2009; Veras et al. 2009) or a resonant migration process (Masset \& Snellgrove 2001; Crida et al. 2009).

\subsubsection{Future Prospects}

At this stage, it is not clear that the properties of transition disks can help differentiate between these planet formation mechanisms - even if it can be confirmed that their structures are produced by planetary-mass companions. However, a future census of companions embedded in such disks that determines the joint distribution of $\left\{a, M_{s}\right\}$ would help guide that debate. In that context, the highest priority should be placed on continuing the high-contrast and high-resolution infrared searches for low-mass companions inside the known transition disk cavities, at the very least to rule out the presence of their more massive (stellar) counterparts (along the lines of Kraus et al. 2011). The identification of any young exoplanet candidates still embedded in their natal disk material would be a landmark discovery for both planet formation and disk evolution. Moreover, those companions would provide youthful touchstones that could help facilitate a better understanding of their much older counterparts, including the Fomalhaut and HR 8799 planets that orbit just inside their own remnant debris disk rings (Kalas et al. 2008; Marois et al. 2008, 2010).

While those companion searches continue, there are two issues related to the planet formation process that can be better addressed with observations of the transition disks themselves. First, the Atacama Large Millimeter Array (ALMA) project starts operations soon and will routinely achieve extraordinary millimeter continuum sensitivity with sub-arcsecond resolution. Those capabilities will permit the first large, unbiased, and direct searches for large $\left(R_{\text {cav }}>10 \mathrm{AU}\right)$ dust-depleted cavities in nearby disks, and should provide a more definitive assessment of transition disk properties across the entire disk mass distribution (see $§ 5.1 .2$ ). These imag- 
ing surveys will provide a first look at the fraction of disks that have formed long-period giant planets within 1-2 Myr, and can be compared with direct imaging exoplanet searches around older stars in the solar neighborhood. As those surveys are building up statistics, a significant effort should be invested in developing our understanding of the structure and contents of the material inside the transition disk cavities. With a focus on individual sources, ALMA will be sensitive enough to provide stringent constraints on the depletion of mm-sized grains and molecular gas in these cavities, and could potentially detect faint emission signals that can be related to the inner disk mass. The residual $880 \mu \mathrm{m}$ signal in the $\mathrm{LkCa} 15$ disk cavity is a tantalizing hint of the prospects for such studies. Coupled with estimates of the cavity size and accretion rate, that information can be used in concert with numerical simulations to help infer the mass and orbital period of the planetary companion perturbing the disk structure. While the present study is just one of the first steps, ultimately high angular resolution radio mapping of disk structures can serve as a new tool to guide searches for young exoplanets, as well as to characterize some of their fundamental properties.

\section{SUMMARY}

We have presented a sample of new and archival SMA observations of the $880 \mu \mathrm{m}$ continuum emission from protoplanetary transition disks at resolution scales down to 0.3 (20-40 AU). Using two-dimensional Monte Carlo radiative transfer calculations and a parametric formulation for the disk densities, we simultaneously modeled these SMA visibilities and the broadband SEDs for each disk in a homogeneous framework. The data and those modeling results were then synthesized to discuss the generic properties of transition disks from a primarily millimeter-wave perspective, as well as to assess the roles of various potential disk evolution mechanisms. Our key results include:

1. In all cases, the SMA data resolve large $\left(R_{\text {cav }}=15\right.$ $73 \mathrm{AU})$, dust-depleted cavities at the disk centers. Unlike estimates based on models of the unresolved infrared spectra, these direct imaging measurements constrain the cavity sizes with little ambiguity: $R_{\text {cav }}$ uncertainties are typically only $\sim 10 \%$ (or up to $15-20 \%$ if a range of surface density profiles are considered).

2. Some dust is still present inside these disk cavities, although its mass and spatial distribution are poorly constrained. With respect to continuous density models, we estimate from the SMA data that the densities of mm-sized particles are $\geq 10$ $100 \times$ lower inside the cavities. From the infrared SED, we find that $\mu \mathrm{m}$-sized particles are depleted by larger factors, typically 4-6 orders of magnitude. Despite these low densities, the dust inside the cavities can have profound effects on the infrared spectrum and the structures we infer at larger radii.

3. Outside the cavities, the transition disk structures appear to be similar to disks with continuous density distributions: they have the same ranges of characteristic radii $\left(R_{c}\right)$ and total masses $\left(M_{d}\right)$.
In their mass reservoirs at large radii, the surface densities for the transition disks are slightly higher than normal disks with similar millimeter luminosities.

4. Our sample is biased toward millimeter-bright disks with large $\left(R_{\text {cav }} \gtrsim 15 \mathrm{AU}\right)$ dust-depleted cavities. However, we find that this specific type of transition disk is surprisingly common among the Taurus/Ophiuchus disks with large millimeter luminosities (or disk masses): they comprise at least $20 \%$ of the disks in the upper half of the $L_{\mathrm{mm}}$ (or $M_{d}$ ) distribution, and $>25 \%$ in the upper quartile. Imaging surveys of other millimeter-bright disks rule out large-cavity transition disk fractions in excess of $70 \%$, but there is no such information available for smaller cavities or fainter disks. The transition disk fractions derived from direct images of cavities are higher than those estimated from infrared colors because the latter technique systematically misses cases with excess emission from a small amount of dust inside the cavity.

5. The large cavity sizes, relatively high accretion rates, and low X-ray luminosities suggest that the transition disk cavities in this sample were not cleared by an internal photoevaporation process (as was already argued by the developers of those dispersal models).

6. If the diminished signatures of dust grains in the cavities are to be explained by particle growth alone, the transition disk structures need to contain very large MRI-inactive dead zones, have experienced growth to at least meter-sized solids, and harbor only very limited reservoirs of small dust grains in their inner disks (from collision fragments or dust coupled to a layered accretion flow). While those stringent conditions make this mechanism for cavity formation seem challenging, a real assessment of the role of particle growth in producing transition disk structures cannot be made until more detailed models are explored.

7. In principle, dynamical interactions with stellar companions on $a \approx 4-40$ AU orbits would be capable of opening the disk cavities we measure. However, recent infrared searches definitively rule out any such companions with mass ratios $q \gtrsim 0.01$ for half of this sample. Moreover, the transition disks studied here have substantially higher (typically $\geq 30 \times$ ) millimeter-wave luminosities (or disk masses) than the disks in multiple star systems with 4-40 AU separations, suggesting that they did not suffer the same level of mass loss from tidal perturbations.

8. We find the best overall agreement with the data if these transition disk cavities are opened by tidal interactions with low mass ratio $(q \gtrsim 0.001)$ companions - either low-mass brown dwarfs or giant planets. Such a companion can open a gap and substantially deplete material interior to its orbit without disrupting the outer disk. We demonstrate 
that the typical accretion rates for these transition disks are decreased (by $\sim 70 \%$ ) compared to their high- $L_{\mathrm{mm}}$ normal disk counterparts, commensurate with the reduced mass flow that is expected to cross the orbit of a low-mass companion (however, a more in-depth analysis with larger samples is warranted). In the near future, radio imaging studies of transition disk structures could provide a new and unique method for identifying and characterizing very young $(\sim 1 \mathrm{Myr})$ low-mass companions - including giant planets on long-period orbits - still embedded in their natal disk material.

We thank Karin Öberg, Sijme-Jan Paardekooper, and Aurélien Crida for beneficial conversations, and a referee for a thorough review that helped clarify the text. We are especially grateful to Adam Kraus, who kindly shared his results prior to publication. The SMA is a joint project between the Smithsonian Astrophysical Observatory and the Academia Sinica Institute of Astronomy and Astrophysics and is funded by the Smithsonian Institution and the Academia Sinica. 


\section{APPENDIX}

\section{COMMENTS ON INDIVIDUAL SOURCES}

This appendix highlights some aspects of the modeling results for individual disks, and compares with previous efforts to characterize their disk cavities whenever available. Table 5 is a compilation of the original sources used to construct the SEDs displayed in Figures 6 and 7.

MWC 758 - - This Herbig Ae star is relatively isolated, located east of the main Taurus-Auriga clouds. Without a firm cluster association, its distance is uncertain but assumed here to be the nominal Hipparcos value, $d=200 \mathrm{pc}$ (Perryman et al. 1997). We adopt the A8 spectral classification advocated by Beskrovnaya et al. (1999), but note that others have utilized an earlier type (A5; van den Ancker et al. 1998). The SMA observations from the E configuration are the same as presented by Isella et al. (2010b), although they have been re-calibrated with an amplitude scale that amounted to a $\sim 30 \%$ decrease in flux levels. That modification was required to reconcile those data and our new SMA observations with short antenna spacings (C configuration); the combined data are now in excellent agreement with single-dish photometry measurements. The $880 \mu \mathrm{m}$ visibility null calls for the largest cavity in the sample, $R_{\text {cav }}=73 \mathrm{AU}$, despite the bright infrared excess. The standard infrared signatures of a transition disk are heavily muted, washed out by only a small amount of dust inside the cavity. Although that dust has densities that are comparable to the other disks in the sample (and only modest rim and cavity heights), it produces a significantly brighter infrared spectrum for two reasons: (1) the central star is extraordinarily hot and luminous, substantially heating more dust, and (2) the scale heights are large, increasing the irradiated surface area and therefore the emitted flux from the disk atmosphere.

Isella et al. (2010b) also modeled the MWC 758 disk structure with some of these SMA data (and supplementary $3 \mathrm{~mm}$ observations), choosing not to modify the accretion disk model to include a cavity (i.e., $\Sigma=\Sigma_{g}$ at all radii in their work) but to allow the gradient of the viscosity profile $(\gamma)$ to vary freely. They can reproduce the data with a large negative viscosity gradient, $\gamma \approx-4$, which results in a surface density profile that increases like $R^{4}$ inside $\sim 70$ AU, peaks near $100 \mathrm{AU}$, and then drops off like $1 / \exp \left(R^{6}\right)$ at larger radii. Roughly speaking, that density distribution is similar to the narrow ring employed here (see Figure 8), even peaking at essentially the same location and surface density. Not unexpectedly, the Isella et al. model requires an additional dust component near the star to account for the infrared excess (which their $R^{4}$ density profile alone vastly under-produces), similar to the inner disk material utilized here. Finally, the sharp density cutoff at large radii that is inherent to models with negative $\gamma$ values means that the Isella et al. model has effectively no mass outside $R \approx 150 \mathrm{AU}$. On the contrary, our model has gas densities in excess of $10^{-5} \mathrm{~g} \mathrm{~cm}^{-2}$ out to $R \approx 400 \mathrm{AU}$, despite the small characteristic radius (because $\gamma=1$ ). We expect that this difference in the effective disk size would help explain the strong positive residuals at large radii noted by Isella et al. when applying their dust model to the $\mathrm{CO}$ emission.

SAO 206462 - - Commonly referred to as HD $135344 \mathrm{~B}$, this star is also isolated, perhaps associated with the outskirts of the Sco-OB2 association. We adopt the distance estimate of van Boekel et al. (2005), $d=140 \mathrm{pc}$, and the F4 spectral type determined by Dunkin et al. (1997). The SMA data were first presented by Brown et al. (2009), and only include observations in the $\mathrm{V}$ configuration with few short baselines. Our models imply a large cavity, $R_{\text {cav }}=46 \mathrm{AU}$, with a small amount of dust near the star. The super-heated inner rim near the dust sublimation radius is responsible for the substantial near-infrared bump in the SED. To minimize the strength of the $10 \mu \mathrm{m}$ silicate feature, comparatively large $\left(s_{\max }=3 \mu \mathrm{m}\right)$ dust grains were used to populate the inner disk. Our model tends to over-predict the flux densities at wavelengths longward of $1 \mathrm{~mm}$, a not uncommon feature due to our decision to fix the grain size distribution in the midplane of the outer disk (see §3.2). The issue might also be alleviated if new data with short antenna spacings become available, enabling a more robust disk mass estimate. The SMA image of the SAO 206462 disk is notably asymmetric, with enhanced emission to the southeast and a pronounced deficit to the north. But the imaged residuals indicate that those asymmetries are only marginally significant: given the challenges of observing such a southern target, those features are not inconsistent with noise (but note that a similar pattern is present in the scattered light images presented by Grady et al. 2009).

Brown et al. (2007) also used RADMC calculations to reproduce the SED alone, and later combined with these SMA data (Brown et al. 2009), but with a different density model (a power-law $\Sigma$ profile, truncated at the edges). They estimated roughly the same cavity radius, 39-45 AU, consistent within the expected $\sim 10 \%$ mutual uncertainties. Although their lower $R_{\text {cav }}$ value was determined from the SMA data, the difference with the cavity size derived here is likely due to small offsets in the adopted disk centroids and viewing geometries. Regarding the latter, we utilize the inclination determined by Lvo et al. (2011) from CO spectral line images, in good agreement with the scattered light constraints of Grady et al. (2009). Those scattered light images suggest a substantially settled vertical distribution of dust with an effective $\psi=-0.3$, unlike the more common $\psi=0.15$ used here. Part of that difference is related to shadowing from the inner rim/disk and cavity wall, which can effectively steepen the scattered light surface brightness profile. But there is so little radial dynamic range available from this disk that it should be possible to find anti-flaring models that are also commensurate with the SMA data.

LkHo 330 - - Associated with the Perseus molecular clouds $(d \approx 250 \mathrm{pc}$; see Enoch et al. 2006), this young star was classified as spectral type G3 by Cohen \& Kuhi (1979). The SMA data in the V configuration were first presented by Brown et al. (2008); new SMA data in the $\mathrm{C}$ configuration are also included here. Note that there is considerable 
disagreement between the IRAS and Spitzer data in the far-infrared, perhaps due to molecular cloud or cirrus contamination in the former. When re-calibrating the long-baseline SMA data used by Brown et al. (2008, 2009), it was noted that the observations of 2006 November 11 had such poor phase stability that they could not be used. With their removal and the addition of new data with short antenna spacings, a much more symmetric and sensitive map of the $880 \mu \mathrm{m}$ continuum emission is available.

We measure a large cavity, $R_{\text {cav }}=68$ AU, $\sim 35-60 \%$ larger than estimated by Brown et al. (2008, 2009) from (some of) the same data. Most of that discrepancy is caused by an inaccurate estimate of the $880 \mu \mathrm{m}$ visibility null location, due to the poor quality of one of the SMA tracks in the $\mathrm{V}$ configuration. Using the CO $J=3-2$ spectral line images from the new data in the $\mathrm{C}$ configuration, we also use an updated viewing geometry (with a significant difference in the major axis PA; the full CO datasets will be presented elsewhere). The faint $10 \mu \mathrm{m}$ silicate feature was difficult to reproduce with the typical grain size index $(p=3.5)$, but we found some success using a more top-heavy distribution $(p=2.5)$ for the dust inside the cavity. To reproduce the relatively bright infrared excess with that grain population, a substantial increase in the inner rim height was required (the dust size distribution parameters and rim height are strongly degenerate; see $\S 3.5)$.

SR 21 - - This young star with spectral type G3 is still embedded in the L1688 dark cloud in the nearby Ophiuchus star-forming region, $d \approx 125$ pc (e.g., Lombardi et al. 2008; Loinard et al. 2008). The extinction estimate of Prato et al. (2003), $A_{V} \sim 9$, is too large to be appropriate for both the optical photometry of Vrba et al. (1993) and the near-infrared magnitudes in the 2MASS catalog (Cutri et al. 2003). Assuming variability is not responsible for that discrepancy, we adopt a lower extinction $\left(A_{V}=6.3\right)$ and therefore a substantially lower $L_{*}$ compared to Brown et al. (2007, 2009). The SMA data are the same as described by Andrews et al. (2009), although the $880 \mu \mathrm{m}$ continuum map has been synthesized with slightly improved resolution. The $880 \mu \mathrm{m}$ continuum emission is distributed in a nearly face-on ring, with $R_{\text {cav }}=36 \mathrm{AU}$. The narrow ring width $\left(R_{c}\right.$ is only $\left.15 \mathrm{AU}\right)$ makes it difficult to determine the viewing geometry, and there is no uncontaminated CO emission or scattered light image available to facilitate an independent measurement. The lack of a clear silicate feature in the mid-infrared leads us to adopt a large $s_{\max }(10 \mu \mathrm{m})$ for the dust inside the cavity. Despite the different modeling setups, the disk structure parameters inferred here are essentially the same as those determined by Andrews et al. (2009). With (some of) the same SMA data, Brown et al. (2009) also find a comparable cavity size (33 AU). We find positive $3 \sigma$ residuals for this model, suggesting excess emission to the southeast and near the stellar position. Those discrepancies lead to a poor prediction of the secondary visibility null (see Figure 6), which may indicate that there is a small amount of $\sim$ mm-sized dust emitting from inside the cavity.

UX Tau $A-$ - The primary star of a wide multiple system in Taurus $(d \approx 140$ pc; see Torres et al. 2009), UX Tau A is the only component with evidence for remnant disk material (e.g., White \& Ghez 2001; McCabe et al. 2006). No $880 \mu \mathrm{m}$ emission above $4.5 \mathrm{mJy}(3 \sigma)$ is detected around either the UX Tau B or C components (in agreement with Jensen \& Akeson 2003). The SMA data presented here are the first resolved observations of the UX Tau A disk. This emission is quite compact: we use a small characteristic radius $\left(R_{c}=20 \mathrm{AU}\right)$, presumably produced by the truncation of the outer disk by UX Tau C (located $\sim 2 . .5$ to the west). Unlike the compact continuum, we find a bright, resolved CO $J=3-2$ disk orbiting UX Tau A, providing the first direct estimates of the disk inclination and position angle. As discussed in $§ 5.1$, the $880 \mu \mathrm{m}$ image shows a resolved cavity that is substantially smaller, $R_{\text {cav }}=25 \mathrm{AU}$, than initial estimates from the SED alone (56 and 71 AU by Espaillat et al. 2007, 2010, respectively). Like LkH $\alpha 330$, we assume a top-heavy dust size distribution $(p=2.5)$ in the cavity to avoid over-producing the $10 \mu \mathrm{m}$ silicate feature.

SR $24 S$ - - Embedded in the L1688 dark cloud in Ophiuchus, SR 24 is a hierarchical triple system with a close binary $\left(0 \prime 2\right.$; Simon et al. 1995) located $\sim 5^{\prime \prime}$ north of a spectral type K2 primary (SR 24 S). The SR 24 N binary exhibits some thermal excess presumably associated with a low-mass circumbinary disk, although it is not detected at millimeter wavelengths (despite evidence for associated CO line emission; Andrews \& Williams 2005a). Due to potential confusion with the SR $24 \mathrm{~N}$ component, we did not use a Spitzer IRS observation of this system (although one apparently exists). The $880 \mu \mathrm{m}$ image of the SR $24 \mathrm{~S}$ disk shows an elongated ring with a significant brightness asymmetry along the major axis (brighter to the north). We measure a moderate cavity size, $R_{\text {cav }}=29 \mathrm{AU}$, essentially the same as our previous effort (32 AU; Andrews et al. 2010b). Naturally, the model does not reproduce the observed asymmetry, instead overpredicting the emission to the southwest (although marginally, based on the imaged residuals). In general, the fit quality here is superior to our initial work, thanks to the modification of the inner rim/disk structure. However, without a detailed infrared spectrum the dust properties and structure of the cavity are uncertain. Unfortunately, the asymmetry of the narrow emission ring makes a clear viewing geometry estimate difficult, and the CO emission from SR $24 \mathrm{~S}$ is contaminated by local cloud material. Substantial improvements in the structure constraints are feasible with a more complete SED, if the disk inclination can be measured independently (e.g., with a tracer like ${ }^{13} \mathrm{CO}$ ).

DoAr 44 - - Sometimes referred to as ROXs 44, this young star is located in the densely-populated L1688 dark cloud in Ophiuchus. A preliminary analysis of the DoAr 44 disk structure was presented by Andrews et al. (2009), using the same SMA data. As in that work, we find a moderate cavity size for the DoAr 44 disk, $R_{\text {cav }}=30$ AU. However, our new model is in much better agreement with the infrared SED, thanks to the inclusion of an inner rim and cavity wall in the model structure. To match the relatively faint far-infrared emission, the dust in the outer disk is substantially concentrated toward the midplane. Unfortunately, there are no independent constraints on the disk inclination or orientation; the adopted inclination could be uncertain by $\sim 20^{\circ}$. The small extent of the $880 \mu$ m emission suggests that the disk is quite small (the ring is narrow), with $R_{c}=25 \mathrm{AU}$. While the western peak of the $880 \mu \mathrm{m}$ 
emission appears slightly brighter, the model residuals show that the asymmetry is not significant. Espaillat et al. (2010) modeled the DoAr 44 SED and derived a slightly larger cavity size (36 AU), but within the uncertainties.

LkCa 15 - - Located in the Taurus star-forming region, this transition disk has been studied extensively since its cavity was first resolved at $1.3 \mathrm{~mm}$ by Piétu et al. (2006). Because of the large spatial extent and prominent depression in the $880 \mu \mathrm{m}$ emission, these new SMA observations make for a spectacular example of a resolved transition disk image. The cavity size derived here, $R_{\text {cav }}=50 \mathrm{AU}$, is in excellent agreement with the initial measurement (46 AU) by Piétu et al. (2006). The SED modeling by Espaillat et al. (2010) argues for a slightly larger cavity (58 AU), although that would be difficult to reconcile with the well-defined $880 \mu \mathrm{m}$ visibility null (see Figure 7 ). The SEDs for the LkCa 15 and DoAr 44 disks are quite similar, with both calling for relatively settled vertical dust distributions. An examination of the modeling results in this case reveals a striking discrepancy with the data: the model predicts far too little millimeter emission inside the cavity (leaving $6 \sigma$ residuals at the stellar position). The integrated residual flux density is small, $\sim 10 \mathrm{mJy}$, but potentially important because it can provide a more direct estimate for the dust mass inside the cavity. For the sake of homogeneity with the rest of this sample, we postpone a more detailed modeling of the LkCa 15 disk cavity until higher resolution images are available.

$R X J 1615$-3255 - - Perhaps less well-known than the other disks in this sample, this system has been kinematically tied to the Lupus association, with $d \approx 185 \mathrm{pc}$ (Makarov 2007). Although it had been identified as a weak-line T Tauri star (Henize 1976; Krautter et al.|1997), infrared observations by Spitzer have only recently claimed RX J1615-3255 as a transition disk candidate (Merín et al. 2010). The new SMA data presented here represent the first spatially resolved images of thermal emission from this disk. A bright, rotating CO $J=3-2$ emission structure is also detected, providing a preliminary estimate of the viewing geometry. Although much of the outer disk emission is spatially filtered in the synthesized $880 \mu \mathrm{m}$ maps shown in Figures 1 and 7, the visibilities clearly indicate that RX J1615-3255 hosts a rather large disk, with $R_{c}=115 \mathrm{AU}$ (in good agreement with the extent of the $\mathrm{CO}$ emission). A particularly low-density cavity is present out to $R_{\text {cav }}=30 \mathrm{AU}$. In fact, we were forced to empty the inner disk inside a radius of $0.5 \mathrm{AU}$ to avoid producing a near-infrared emission signature above photospheric levels (therefore, no inner rim was employed in this model; see Table 4). To reproduce the comparatively faint infrared excess beyond the dip in the SED, the scale heights were decreased to values consistent with substantial dust settling. The resulting low dust temperatures, coupled with the large disk size, lead to a relatively high estimate for the total disk mass $\left(\sim 0.13 \mathrm{M}_{\odot}\right.$, almost $12 \%$ of the stellar mass).

GM Aur - _ This star in the Taurus star-forming region is perhaps the canonical example of a transition disk, with many detailed discussions of the implications of its distinctive infrared SED (e.g., Strom et al. 1989; Skrutskie et al. 1990; Koerner et al. 1993; Chiang \& Goldreich 1999; Rice et al. 2003a). Using some of the same SMA data, Hughes et al. (2009a) first resolved the central dust cavity. We include new $880 \mu \mathrm{m}$ SMA observations here, with short (C) and intermediate (E) antenna spacings that provide a substantially improved Fourier sampling near the visibility null. The cavity size derived here, $R_{\text {cav }}=28 \mathrm{AU}$, is larger than the preferred Hughes et al. (2009a) value (20 AU) because the new SMA observations provide a more accurate location for the $880 \mu \mathrm{m}$ visibility null (at a slightly shorter deprojected baseline). However, the alternative disk model discussed by Hughes et al. (2009a) employed a larger cavity (26 AU) that is in good agreement with our model. The long-baseline SMA data is quite noisy in this case; improved sensitivity would help provide a more accurate cavity size estimate. Like the Hughes et al. (2009a) results, our model successfully matches a high resolution $1.3 \mathrm{~mm}$ dataset from the Plateau de Bure interferometer. The GM Aur disk is also found to be quite large, with a characteristic size $R_{c}=120 \mathrm{AU}$ (although most of that emission has been filtered out in the images displayed here; see Table 2).

DM Tau - _ Although the gas disk around this cool, young star in the Taurus clouds has been studied extensively (e.g., Guilloteau \& Dutrev 1998; Dartois et al. 2003; Piétu et al. 2007), our new SMA observations permit the first sub-arcsecond resolution examination of the $880 \mu \mathrm{m}$ dust continuum emission. Based on the shape of its Spitzer IRS spectrum, Calvet et al. (2005) suggested that the DM Tau disk has a relatively small cavity of radius $\sim 3$ AU. Since such a small cavity cannot be resolved on the longest SMA baselines, it was a surprise to measure a clear $880 \mu \mathrm{m}$ emission ring and visibility null indicative of a much larger dust-depleted cavity, $R_{\text {cav }}=19$ AU. Reconciling this large cavity size in the SMA data with the morphology of the IRS spectrum was a challenge, and our current model should be considered preliminary until the latter data is better matched. To account for the absence of an infrared excess shortward of $\sim 8 \mu \mathrm{m}$, we were forced to remove all dust inside a radius of $1 \mathrm{AU}$ (there is no inner rim in this case; see Table 4). Like the cases of GM Aur and LkCa 15, we find that the DM Tau disk is rather large, with $R_{c}=135 \mathrm{AU}$, in good agreement with the extended CO emission studied elsewhere.

WSB 60 - - The dust-depleted cavity in the disk around this very cool star (spectral type M4) in the core of the Ophiuchus star-forming region was first discovered by Andrews et al. (2009). Compared to that initial study, we find a slightly smaller cavity here $\left(R_{\text {cav }}=15 \mathrm{AU}\right.$, compared to $\left.20 \mathrm{AU}\right)$, partly related to our choice to fix the surface density gradient to $\gamma=1$ in the new models. The cavity is just barely resolved with our SMA observations, and it shows no obvious signatures of dust depletion at infrared wavelengths. The latter point drives us to infer the smallest dust depletion factor inside the cavity for this sample, corresponding to only a factor of $\sim 50 \times$ lower densities in the inner disk than the nominal continuous model. Considering the "normal" appearance of the infrared SED, it is natural to speculate that there are potentially many other such disks that have similarly large cavities but will only be identified when high resolution radio images are available. 
Table 5

Sources for SED Data

\begin{tabular}{lcccc}
\hline \hline \multicolumn{1}{c}{$\begin{array}{c}\text { Name } \\
(1)\end{array}$} & $\begin{array}{c}\text { optical } \\
(2)\end{array}$ & $\begin{array}{c}\text { infrared } \\
(3)\end{array}$ & $\begin{array}{c}\text { Spitzer } \text { IRS } \\
(4)\end{array}$ & $\begin{array}{c}\text { (sub)-mm } \\
(5)\end{array}$ \\
\hline MWC 758 & 1 & $1,2,3$ & 1 & 1,2 \\
SAO 206462 & 2 & $1,4,5$ & 2 & $3,4,5$ \\
LkH $\alpha$ 330 & 3 & $1,3,6,7$ & 3 & $5,6,7,8$ \\
SR 21 & 4 & $1,3,6$ & 4 & $9,10,11$ \\
UX Tau A & 5 & $1,3,8,9$ & 5 & 12,13 \\
SR 24 S & 6,7 & $1,6,9,10$ & $\cdots$ & $9,10,11$ \\
DoAr 44 & 8 & $1,6,11$ & 4 & 9,14 \\
LkCa 15 & 9 & 1,12 & 5 & $12,15,16,17,18$ \\
RX J1615-3255 & 10 & $1,3,13$ & 6 & 19 \\
GM Aur & 11 & $1,3,8$ & 7 & $12,17,20,21,22,23,24$ \\
DM Tau & 5 & $1,3,8$ & 7 & $17,25,26$ \\
WSB 60 & 7 & $1,6,11$ & 4 & 9 \\
& & & & \\
\hline
\end{tabular}

Note. - Col. (1): Name of host star. Col (2): Optical references: [1] Vieira et al. (2003), [2] - Coulson \& Walther (1995), [3] - Fernández \& Eiroa (1996), [4] - Vrba et al. (1993), [5] - Kenyon \& Hartmann (1995), [6] - Herbig \& Bell (1988), [7] - Wilking et al. (2005), [8] - Herbst et al. (1994), [9] - Bouvier et al. (1993), [10] - Makarov (2007), [11] - Bouvier et al. (1995) Col. (3): Infrared references: [1] 2MASS (Cutri et al. 2003), [2] - Malfait et al. (1998), [3] - IRAS (Beichman et al. 1988), [4] - Coulson \& Walther (1995), [5] - Walker \& Wolstencraft (1988), [6] Evans et al. (2003), [7] - Rebull et al. (2007), [8] - Luhman et al. (2010), [9] McCabe et al. (2006), [10] - Jensen \& Mathieu (1997), [11] - Padgett et al. (2008), [12] - Rebull et al. (2010), [13] - Padgett et al. (2006), Col. (4): Spitzer IRS spectrum references: [1] - the Spitzer archive, reduced as in McClure et al. (2010), [2] - Brown et al. (2007), [3] - Brown et al. (2008), [4] - McClure et al. (2010), [5] Espaillat et al. (2007), [6] - Evans et al. (2003), [7] - Calvet et al. (2005) Col. (5): Millimeter references: [1] - Di Francesco et al. (2008), [2] - Chapillon et al. (2008), [3] - Coulson \& Walther (1995), [4] - Svlvester et al. (1996), [5] - Brown et al. (2009), [6] - Brown et al. (2008), [7] - Osterloh \& Beckwith (1995), [8] - Enoch et al. (2006), [9] Andrews \& Williams (2007b), [10] - André \& Montmerle (1994), [11] - Patience et al. (2008), [12] - Andrews \& Williams (2005b), [13] - Jensen \& Akeson (2003), [14] Nürnberger et al. (1998), [15] - Beckwith et al. (1990), [16] - Duvert et al. (2000), [17] - Kitamura et al. (2002), [18] - Piétu et al. (2006), [19] - Lommen et al. (2010), [20] - Weintraub et al. (1989), [21] - Beckwith \& Sargent (1991), [22] - Dutrev et al. (1998), [23] - Loonev et al. (2000), [24] - Hughes et al. (2009a), [25] - Isella et al. (2009), [26] - Guilloteau \& Dutrey (1998). 
REFERENCES

Abt, H. A., Levy, S. G. 1976, ApJS, 30, 273

Aikawa, Y., \& Nomura, H. 2006, ApJ, 642, 1152

Alexander, R. D., Clarke, C. J., \& Pringle, J. E. 2004, MNRAS, 348, 879

Alexander, R. D., Clarke, C. J., \& Pringle, J. E. 2005, MNRAS, 358, 283

Alexander, R. D., Clarke, C. J., \& Pringle, J. E. 2006, MNRAS, 369, 216 (2006a)

Alexander, R. D., Clarke, C. J., \& Pringle, J. E. 2006, MNRAS, 369, 229 (2006b)

Alexander, R. D., \& Armitage, P. J. 2007, MNRAS, 375, 500

Alexander, R. D., \& Armitage, P. J. 2009, ApJ, 704, 989

Alibert, Y., Mordasini, C., Benz, W., \& Winisdoerffer, C. 2005, A\&A, 434, 343

André, P., \& Montmerle, T. 1994, ApJ, 420, 837

Andrews, S. M., \& Williams, J. P. 2005, ApJ, 619, L175 (2005a)

Andrews, S. M., \& Williams, J. P. 2005, ApJ, 635, 1134 (2005b)

Andrews, S. M., \& Williams, J. P. 2007, ApJ, 659, 705 (2007a)

Andrews, S. M., \& Williams, J. P. 2007, ApJ, 671, 1800 (2007b)

Andrews, S. M., Wilner, D. J., Hughes, A. M., Qi, C., \& Dullemond, C. P. 2009, ApJ, 700, 1502

Andrews, S. M., Czekala, I., Wilner, D. J., Espaillat, C., Dullemond, C. P., \& Hughes, A. M. 2010, ApJ, 710, 462 (2010a)

Andrews, S. M., Wilner, D. J., Hughes, A. M., Qi, C., \& Dullemond, C. P. 2010, ApJ, 723, 1241 (2010b)

Artymowicz, P., \& Lubow, S. H. 1994, ApJ, 421, 651

Artymowicz, P., \& Lubow, S. H. 1996, ApJ, 467, L77

Balbus, S. A., \& Hawley, J. F. 1991, ApJ, 376, 214

Barsony, M., Koresko, C., \& Matthews, K. 2003, ApJ, 591, 1064

Beckwith, S. V. W., Sargent, A. I., Chini, R. S., \& Güsten, R. 1990, AJ, 99, 924

Beckwith, S. V. W., \& Sargent, A. I. 1991, ApJ, 381, 250

Beichman, C. A., Neugebauer, G., Habing, H. J., Clegg, P. E., \& Chester, T. J. (ed.) 1988, Infrared Astronomical Satellite (IRAS) Catalogs and Atlases, Vol. 1: Explanatory Supplement

Beskrovnaya, N. G., et al. 1999, A\&A, 343, 163

Beust, H., \& Dutrey, A. 2005, A\&A, 439, 585

Birnstiel, T., Dullemond, C. P., \& Brauer, F. 2010, A\&A, 513, 79

Birnstiel, T., Ormel, C. W., \& Dullemond, C. P. 2011, A\&A, 525, 11

Boley, A. C. 2009, ApJ, 695, L53

Bouvier, J., Cabrit, S., Fernández, M., Martín, E. L., \& Matthews, J. M. 1993, A\&A, 272, 176

Bouvier, J., et al. 1995, A\&A, 299, 89

Boss, A. P. 1997, Science, 276, 1836

Boss, A. P. 1998, ApJ, 503, 923

Boss, A. P. 2006, ApJ, 637, L137

Boss, A. P. 2009, ApJ, 694, 107

Brauer, F., Dullemond, C. P., \& Henning, T. 2008, A\&A, 480, 859

Brown, J. M., et al. 2007, ApJ, 664, L107

Brown, J. M., Blake, G. A., Qi, C., Dullemond, C. P., \& Wilner, D. J. 2008, ApJ, 675, L109

Brown, J. M., Blake, G. A., Qi, C., Dullemond, C. P., Wilner, D. J., \& Williams, J. P. 2009, ApJ, 704, 496

Bryden, G., Chen, X., Lin, D. N. C., Nelson, R. P., \& Papaloizou, J. C. B. 1999, ApJ, 514, 344

Calvet, N., D’Alessio, P., Hartmann, L., Wilner, D., Walsh, A., \& Sitko, M. 2002, ApJ, 568, 1008

Calvet, N., et al. 2005, ApJ, 630, L185

Chapillon, E., Guilloteau, S., Dutrey, A., \& Piétu, V. 2008, A\&A, 488, 565

Chiang, E. I., \& Goldreich, P. 1999, ApJ, 519, 279

Chiang, E. I., et al. 2001, ApJ, 547, 1077

Cieza, L. A., et al. 2007, ApJ, 667, 308

Cieza, L. A., Swift, J. J., Mathews, G. S., \& Williams, J. P. 2008, ApJ, 688, L115

Cieza, L. A., et al. 2009, ApJ, 696, L84

Cieza, L. A., et al. 2010, ApJ, 712, 925

Clarke, C. J., Gendrin, A., \& Sotomayor, M. 2001, MNRAS, 328, 485

Clarke, C. J. 2009, MNRAS, 396, 1066

Cohen, M., \& Kuhi, L. V. 1979, ApJS, 41, 743

Coulson, I. M., \& Walther, D. M. 1995, MNRAS, 274, 977

Crida, A., Morbidelli, A., \& Masset, F. 2006, Icarus, 181, 587

Crida, A., \& Morbidelli, A. 2007, A\&A, 377, 1324

Crida, A., Masset, F., \& Morbidelli, A. 2009, ApJ, 705, L148

Cutri, R. M., et al. 2003, 2MASS All-Sky Point Source Catalog (Pasadena: IPAC)

Currie, T., Lada, C. J., Plavchan, P., Robitaille, T. P., Irwin, J., \& Kenyon, S. J. 2009, ApJ, 698, 1 (2009a)

Currie, T., \& Kenyon, S. J. 2009, AJ, 138, 703 (2009b)

D’Alessio, P., Calvet, N., Hartmann, L., Lizano, S., \& Cantó, J. 1999, ApJ, 527, 893

D'Alessio, P., Calvet, N., \& Hartmann, L. 2001, ApJ, 553, 321

D'Alessio, P. et al. 2005, ApJ, 621, 461

D'Alessio, P., Calvet, N., Hartmann, L., Franco-Hernández, R., \& Servín, H. 2006, ApJ, 638, 314

Damiani, F., Micela, G., Sciortino, S., \& Harnden, F. R. 1995, ApJ, 446, 331

Dartois, E., Dutrey, A., \& Guilloteau, S. 2003, A\&A, 399, 773

Di Francesco, J., Johnstone, D., Kirk, H., MacKenzie, T., \& Ledwosinska, E. 2008, ApJS, 175, 277

Dodson-Robinson, S. E., Veras, D., Ford, E. B., \& Beichman, C. A. 2009, ApJ, 707, 79

Draine, B. T. 2006, ApJ, 636, 1114

Draine, B. T., \& Lee, H. M. 1984, ApJ, 285, 89

Drake, J. J., Ercolano, B., Flaccomio, E., \& Micela, G. 2009, ApJ, 699, L35

Ducourant, C., Teixeira, R., Perie, J.-P., Le Campion, J.-F., Guibert, J., \& Sartori, M. J. 2005, A\&A, 438, 769

Dullemond, C. P. 2000, A\&A, 361, L17

Dullemond, C. P., Dominik, C., \& Natta, A. 2001, ApJ, 560, 957 
Dullemond, C. P., van den Ancker, M. E., Acke, B., \& van Boekel, R. 2003, ApJ, 594, L47

Dullemond, C. P., \& Dominik, C. 2004, A\&A, 417, 159 (2004a)

Dullemond, C. P., \& Dominik, C. 2004, A\&A, 421, 1075 (2004b)

Dullemond, C. P., \& Dominik, C. 2005, A\&A, 434, 971

Dunkin, S. K., Barlow, M. J., \& Ryan, S. G. 1997, MNRAS, 290, 165

Duquennoy, A., \& Mayor, M. 1991, A\&A, 248, 485

Dutrey, A., et al. 1998, A\&A, 338, L63

Dutrey, A., et al. 2008, A\&A, 490, L15

Duvert, G., Guilloteau, S., Ménard, F., Simon, M., \& Dutrey, A. 2000, A\&A, 355, 165

Eisner, J. A., Graham, J. R., Akeson, R. L., \& Najita, J. 2009, ApJ, 692, 309

Enoch, M. L., et al. 2006, ApJ, 638, 293

Ercolano, B., Drake, J. J., Raymond, J. C., \& Clarke, C. J. 2008, ApJ, 688, 398

Ercolano, B., Clarke, C. J., \& Drake, J. J. 2009, ApJ, 699, 1639

Espaillat, C., et al. 2007, ApJ, 670, L135

Espaillat, C., et al. 2010, ApJ, 717, 441

Espaillat, C., et al. 2011, ApJ, 728, 49

Evans, N. J., et al. 2003, PASP, 115, 965

Fang, M., et al. 2009, A\&A, 504, 461

Feigelson, E. D., \& Nelson, P. I. 1985, ApJ, 293, 192

Fernández, M., \& Eiroa, C. 1996, A\&A, 310, 143

Flaherty, K. M., \& Muzerolle, J. 2010, ApJ, 719, 1733

Fromang, S., Terquem, C., \& Balbus, S. A. 2002, MNRAS, 329, 18

Furlan, E., et al. 2007, ApJ, 664, 1176

Gammie, C. J. 1996, ApJ, 457, 355

Garaud, P. 2007, ApJ, 671, 2091

Goldreich, P., \& Tremaine, S. 1978, ApJ, 222, 850

Goldreich, P., \& Tremaine, S. 1980, ApJ, 241, 425

Gorti, U., Dullemond, C. P., \& Hollenbach, D. 2009, ApJ, 705, 1237

Grady, C. A., et al. 2009, ApJ, 699, 1822

Grosso, N., Montmerle, T., Bontemps, S., André, P., \& Feigelson, E. D. 2000, A\&A, 359, 113

Guenther, E. W., et al. 2007, A\&A, 467, 1147

Guilloteau, S., \& Dutrey, A. 1998, A\&A, 339, 467

Guilloteau, S., Dutrey, A., \& Simon, M. 1999, A\&A, 348, 570

Hartmann, L., Calvet, N., Gullbring, E., \& D'Alessio, P. 1998, ApJ, 495, 385

Henize, K. G. 1976, ApJS, 30, 491

Herbig, G. H., \& Bell, K. R. 1988, in Third Catalog of Emission Line Stars of the Orion Population (Santa Cruz: Lick Obs.)

Herbst, W., Herbst, D. K., \& Grossman, E. J. 1994, AJ, 108, 1906

Ho, P. T. P., Moran, J. M., \& Lo, K. Y. 2004, ApJ, 616, L1

Høg, E., et al. 1998, A\&A, 335, L65

Hollenbach, D., Johnstone, D., Lizano, S., \& Shu, F. 1994, ApJ, 428, 654

Horedt, G. P. 1978, A\&A, 64, 173

Hubickyj, O., Bodenheimer, P., \& Lissauer, J. J. 2005, Icarus, 179, 415

Hughes, A. M., Wilner, D. J., Calvet, N., D’Alessio, P., Claussen, M. J., \& Hogerheijde, M. R. 2007, ApJ, 664, 536

Hughes, A. M., et al. 2009, ApJ, 698, 131 (2009a)

Hughes, A. M., et al. 2009, ApJ, 704, 1204 (2009b)

Hughes, A. M., et al. 2010, AJ, 140, 887

Hughes, A. M., Wilner, D. J., Andrews, S. M., Qi, C., \& Hogerheijde, M. R. 2010, ApJ, 727, 85

Ida, S., \& Lin, D. N. C. 2004, ApJ, 604, 388

Ida, S., \& Lin, D. N. C. 2005, ApJ, 626, 1045

Ingleby, L., et al. 2009, ApJ, 703, L137

Ireland, M. J., \& Kraus, A. L. 2008, ApJ, 678, L59

Isella, A., Carpenter, J. M., \& Sargent, A. I. 2009, ApJ, 701, 260

Isella, A., Carpenter, J. M., \& Sargent, A. I. 2010, ApJ, 714, 1746 (2010a)

Isella, A., Natta, A., Wilner, D., Carpenter, J. M., \& Testi, L. 2010, ApJ, 725, 1735 (2010b)

Isobe, T., Feigelson, E. D., \& Nelson, P. I. 1986, ApJ, 306, 490

Jensen, E. L. N., Mathieu, R. D., \& Fuller, G. A. 1994, ApJ, 429, L29

Jensen, E. L. N., Mathieu, R. D., \& Fuller, G. A. 1996, ApJ, 458, 312

Jensen, E. L. N., \& Mathieu, R. D. 1997, AJ, 114, 301

Jensen, E. L. N., \& Akeson, R. L. 2003, ApJ, 584, 875

Jonkheid, B., Dullemond, C. P., Hogerheijde, M. R., \& van Dishoeck, E. F. 2007, A\&A, 463, 203

Kalas, P., et al. 2008, Science, 322, 1345

Kenyon, S. J., \& Hartmann, L. 1995, ApJS, 101, 117

Kim, K. H., et al. 2009, ApJ, 700, 1017

Kitamura, Y., Momose, M., Yokogawa, S., Kawabe, R., Tamura, M., \& Ida, S. 2002, ApJ, 581, 357

Kley, W. 1999, MNRAS, 303, 696

Koerner, D. W., Sargent, A. I., \& Beckwith, S. V. W. 1993, Icarus, 106, 2

König, B., Neuhäuser, R., \& Stelzer, B. 2001, A\&A, 369, 971

Kraus, A. L., Ireland, M. J., Martinache, F., \& Hillenbrand, L. A. 2011, ApJ, in press

Kratter, K. M., Murray-Clay, R. A., \& Youdin, A. N. 2010, ApJ, 710, 1375

Krautter, J., Wichmann, R., Schmitt, J. H. M. M., Alcala, J. M., Neuhauser, R., \& Terranegra, L. 1997, A\&AS, 123, 329

Lada, C. J., et al. 2006, AJ, 131, 1574

Lejeune, T., Cuisinier, F., \& Buser, R. 1997, A\&AS, 125, 229

Lin, D. N. C., \& Papaloizou, J. C. B. 1979, MNRAS, 186, 789 (1979a)

Lin, D. N. C., \& Papaloizou, J. C. B. 1979, MNRAS, 188, 171 (1979b)

Lin, D. N. C., \& Papaloizou, J. C. B. 1986, ApJ, 307, 395

Lin, D. N. C., \& Papaloizou, J. C. B. 1993, in Protostars \& Planets III, eds. E. Levy \& M. S. Matthews (Tucson: Univ. Arizona Press), 749 Loinard, L., Torres, R. M., Mioduszewski, A. J., \& Rodríguez, L. F. 2008, ApJ, 675, L29 
Lombardi, M., Lada, C. J., \& Alves, J. 2008, A\&A, 480, L785

Lommen, D. J. P., et al. 2010, A\&A, 515, 77

Looney, L. W., Mundy, L. G., \& Welch, W. J. 2000, ApJ, 529, 477

Lubow, S. H., Seibert, M., \& Artymowicz, P. 1999, ApJ, 526, 1001

Lubow, S. H., \& D'Angelo, G. 2006, ApJ, 641, 526

Luhman, K. L. 1999, ApJ, 525, 466

Luhman, K. L., \& Rieke, G. H. 1999, ApJ, 525, 440

Luhman, K. L., Allen, P. R., Espaillat, C., Hartmann, L., \& Calvet, N. 2010, ApJS, 186, 111

Lynden-Bell, D., \& Pringle, J. E. 1974, MNRAS, 168, 603

Lyo, A.-R., et al. 2011, in preparation

Makarov, V. V. 2007, ApJ, 658, 480

Malfait, K., Bogaert, E., \& Waelkens, C. 1998, A\&A, 331, 211

Marley, M. S., Fortney, J. J., Hubickyj, O., Bodenheimer, P., \& Lissauer, J. J. 2007, ApJ, 655, 541

Marois, C., et al. 2008, Science, 322, 1348

Marois, C., Zuckerman, B., Konopacky, Q. M., Macintosh, B., \& Barman, T. 2010, Nature, 468, 1080

Masset, F., \& Snellgrove, M. 2001, MNRAS, 320, L55

Mathis, J. S. 1990, ARA\&A, 28, 37

Matsumura, S., \& Pudritz, R. E. 2003, ApJ, 598, 645

Matsuyama, I., Johnstone, D., \& Hollenbach, D. 2009, ApJ, 700, 10

McCabe, C., Ghez, A. M., Prato, L., Duchene, G., Fischer, R. S., \& Telesco, C. 2006, ApJ, 636, 932

McClure, M. 2009, ApJ, 693, L81

McClure, M. K., et al. 2010, ApJS, 188, 75

Merín, B., et al. 2010, ApJ, 718, 1200

Miyake, K., \& Nakagawa, Y. 1993, Icarus, 106, 20

Montmerle, T., Koch-Miramond, L., Falgarone, E., \& Grindlay, J. E. 1983, ApJ, 269, 182

Mordasini, C., Alibert, Y., \& Benz, W. 2009, A\&A, 501, 1139

Muzerolle, J., Allen, L. E., Megeath, S. T., Hernández, J., \& Gutermuth, R. A. 2010, ApJ, 708, 1107

Natta, A., Testi, L., \& Randich, S. 2006, A\&A, 452, 245

Najita, J., Carr, J. S., \& Mathieu, R. D. 2003, ApJ, 589, 931

Najita, J. R., Strom, S. E., \& Muzerolle, J. 2007, MNRAS, 378, 369

Najita, J. R., et al. 2010, ApJ, 712, 274

Natta, A., Prusti, T., Neri, R., Wooden, D., Grinin, V. P., \& Mannings, V. 2001, A\&A, 371, 186

Neuhauser, R., Sterzik, M. F., Schmitt, J. H. M. M., Wichmann, R., \& Krautter, J. 1995, A\&A, 297, 391

Nürnberger, D., Brandner, W., Yorke, H. W., \& Zinnecker, H. 1998, A\&A, 330, 549

Osterloh, M., \& Beckwith, S. V. W. 1995, ApJ, 439, 288

Owen, J. E., Ercolano, B., Clarke, C. J., \& Alexander, R. D. 2010, MNRAS, 401, 1415

Owen, J. E., Ercolano, B., \& Clarke, C. J. 2011, MNRAS, in press (arXiv:1010.1079)

Paardekooper, S.-J., \& Mellema, G. 2006, A\&A, 435, 1129

Padgett, D. L., et al. 2006, ApJ, 645, 1283

Padgett, D. L., et al. 2008, ApJ, 672, 1013

Papaloizou, J. C. B., Nelson, R. P., Kley, W., Masset, F. S., \& Artymowicz, P. 2007, in Protostars \& Planets V, eds. B. Reipurth, D. Jewitt, \& K. Keil (Tucson: Univ. Arizona Press), 655

Pascucci, I., \& Sterzik, M. 2009, ApJ, 702, 724

Patience, J., Akeson, R. L., \& Jensen, E. L. N. 2008, ApJ, 677, 616

Perryman, M. A. C., et al. 1997, A\&A, 323, L49

Pichardo, B., Sparke, L. S., \& Aguilar, L. A. 2008, MNRAS, 391, 815

Piétu, V., Dutrey, A., Guilloteau, S., Chapillon, E., \& Pety, J. 2006, A\&A, 460, L43

Piétu, V., Dutrey, A., \& Guilloteau, S. 2007, A\&A, 467, 163

Pillitteri, I., et al. 2010, A\&A, 519, 34

Pollack, J. B., Hubickyj, O., Bodenheimer, P., Lissauer, J. J., Podolak, M., \& Greenzweig, Y. 1996, Icarus, 124,62

Pontoppidan, K. M., Blake, G. A., van Dishoeck, E. F., Smette, A., Ireland, M. J., \& Brown, J. 2008, ApJ, 684, 1323

Pontoppidan, K. M., Salyk, C., Blake, G. A., Meijerink, R., Carr, S., \& Najita, J. 2010, ApJ, 720, 887

Pott, J.-U., Perrin, M., Furlan, E., Ghez, A. M., Herbst, T. M., \& Metchev, S. 2010, ApJ, 710, 265

Prato, L., Greene, T. P., \& Simon, M. 2003, ApJ, 584, 853

Pringle, J. E. 1981, ARA\&A, 19, 137

Quillen, A. C., Blackman, E. G., Frank, A., \& Varniére, P. 2004, ApJ, 612, L137

Rafikov, R. R. 2009, ApJ, 704, 281

Rafikov, R. R. 2011, ApJ, 727, 86

Rebull, L. M., et al. 2007, ApJS, 171, 447

Rebull, L. M., et al. 2010, ApJS, 186, 259

Rice, W. K. M., Wood, K., Armitage, P. J., Whitney, B. A., \& Bjorkman, J. E. 2003, MNRAS, 342, 79 (2003a)

Rice, W. K. M., Armitage, P. J., Bonnell, I. A., Bate, M. R., Jeffers, S. V., \& Vine, S. G. 2003, MNRAS, 346, L36 (2003b)

Rice, W. K. M., Armitage, P. J., Wood, K., \& Lodato, G. 2006, MNRAS, 373, 1619

Salyk, C., Blake, G. A., Boogert, A. C. A., \& Brown, J. M. 2007, ApJ, 655, L105

Salyk, C., Blake, G. A., Boogert, A. C. A., \& Brown, J. M. 2009, ApJ, 699, 330

Sano, T., Miyama, S. M., Umebayashi, T., \& Nakano, T. 2000, ApJ, 543, 486

Scharf, C., \& Menou, K. 2009, ApJ, 693, L113

Shakura, N. I., \& Sunyaev, R. A. 1973, A\&A, 24, 337

Shu, F. H., Johnstone, D., \& Hollenbach, D. 1993, Icarus, 106, 92

Sicilia-Aguilar, A., Henning, T., \& Hartmann, L. 2010, ApJ, 710, 597

Siess, L., Dufour, E., \& Forestini, M. 2000, A\&A, 358, 593

Simon, M., et al. 1995, ApJ, 443, 625

Skrutskie, M. F., Dutkevitch, D., Strom, S. E., Edwards, S., Strom, K. M., \& Shure, M. A. 1990, AJ, 99, 1187

Strom, K. M., Strom, S. E., Edwards, S., Cabrit, S., \& Skrutskie, M. F. 1989, AJ, 97, 1451

Suzuki, T. K., \& Inutsuka, S. 2009, ApJ, 691, L49

Suzuki, T. K., Muto, T., \& Inutsuka, S. 2010, ApJ, 718, 1289

Sylvester, R. J., Skinner, C. J., Barlow, M. J., \& Mannings, V. 1996, MNRAS, 279, 915 
Tanaka, H., Himeno, Y., \& Ida, S. 2005, ApJ, 625, 414

Torres, R. M., Loinard, L., Mioduszewski, A. J., \& Rodríguez, L. F. 2009, ApJ, 698, 242

Uchida, K. I., et al. 2004, ApJS, 154, 439

van Boekel, R., et al. 2005, A\&A, 437, 189

van den Ancker, M. E., de Winter, D., \& Tjin A Djie, H. R. E. 1998, A\&A, 330, 145

Varniére, P., Blackman, E. G., Frank, A., \& Quillen, A. C. 2006, ApJ, 640, 1110

Vasyunin, A. I., Wiebe, D. S., Birnstiel, T., Zhukovska, S., Henning, T., \& Dullemond, C. P. 2011, ApJ, 727,76

Veras, D., Crepp, J. R., \& Ford, E. B. 2009, ApJ, 696, 1600

Vieira, S. L. A., et al. 2003, AJ, 126, 2971

Vrba, F. J., Coyne, G. V., \& Tapia, S. 1993, AJ, 105, 1010

Walker, H. J., \& Wolstencraft, R. D. 1988, PASP, 100, 1509

Weidenschilling, S. J. 1977, MNRAS, 180, 57 (1977a)

Weidenschilling, S. J. 1977, Ap\&SS, 51, 153 (1977b)

Weingartner, J. C., \& Draine, B. T. 2001, ApJ, 548, 296

Weintraub, D. A., Sandell, G., \& Duncan, W. D. 1989, ApJ, 340, L69

White, R. J., \& Ghez, A. M. 2001, ApJ, 556, 265

Wichmann, R., Covino, E., Alcala, J. M., Krautter, J., Allain, S., \& Hauschildt, P. H. 1999, A\&A, 320, 185

Wilking, B. A., Meyer, M. R., Robinson, J. G., \& Greene, T. P. 2005, AJ, 130, 1733

Winters, W. F., Balbus, S. A., \& Hawley, J. F. 2003, ApJ, 589, 543

Zhu, Z., Nelson, R. P., Hartmann, L., Espaillat, C., \& Calvet, N. 2011, ApJ, 729, 47 